Acta Crystallographica Section D

\section{Biological \\ Crystallography}

ISSN 0907-4449

Walter M. Kim, Alexander B. Sigalov and Lawrence J. Stern*

University of Massachusetts Medical School, USA

Correspondence e-mail: lawrence.stern@umassmed.edu

\title{
Pseudo-merohedral twinning and noncrystallographic symmetry in orthorhombic crystals of SIVmac239 Nef core domain bound to different-length TCR $\zeta$ fragments
}

HIV/SIV Nef mediates many cellular processes through interactions with various cytoplasmic and membrane-associated host proteins, including the signalling $\zeta$ subunit of the T-cell receptor $(\mathrm{TCR} \zeta)$. Here, the crystallization strategy, methods and refinement procedures used to solve the structures of the core domain of the SIVmac239 isolate of Nef $\left(\mathrm{Nef}_{\text {core }}\right)$ in complex with two different TCR $\zeta$ fragments are described. The structure of SIVmac239 $\mathrm{Nef}_{\text {core }}$ bound to the longer TCR $\zeta$ polypeptide (Leu51-Asp93) was determined to $3.7 \AA$ resolution $\left(R_{\text {work }}=28.7 \%\right)$ in the tetragonal space group $P 4_{3} 2{ }_{1} 2$. The structure of SIVmac239 $\mathrm{Nef}_{\text {core }}$ in complex with the shorter TCR $\zeta$ polypeptide (Ala63-Arg80) was determined to $2.05 \AA$ resolution $\left(R_{\mathrm{work}}=17.0 \%\right)$, but only after the detection of nearly perfect pseudo-merohedral crystal twinning and proper assignment of the orthorhombic space group $P 2_{1} 2_{1} 2_{1}$. The reduction in crystal space-group symmetry induced by the truncated TCR $\zeta$ polypeptide appears to be caused by the rearrangement of crystal-contact hydrogen-bonding networks and the substitution of crystallographic symmetry operations by similar noncrystallographic symmetry (NCS) operations. The combination of NCS rotations that were nearly parallel to the twin operation $(k, h,-l)$ and $a$ and $b$ unit-cell parameters that were nearly identical predisposed the $P 2{ }_{1} 2_{1} 2_{1}$ crystal form to pseudo-merohedral twinning.

\section{Introduction}

Protein crystallization occurs under supersaturating conditions where protein molecules organize by either noncrystallographic or crystallographic symmetry operations into repeating unit cells that pack to form a crystal lattice. Crystal twinning occurs when two or more crystal packings intersperse in one larger aggregate crystal. This has been reported to occur as a result of polymorphic transformation during physical stress (Yeates, 1997; Govindasamy et al., 2004), but more commonly occurs as a pathology of crystal growth. When the lattices of each crystal packing in the aggregate crystal do not overlap in three dimensions, the crystal exhibits epitaxial, or nonmerohedral, twinning, which can easily be detected by the presence of split reflections in the crystal's X-ray diffraction pattern. However, when the lattice axes of the individual crystals are parallel the crystal is considered to be merohedrally twinned and the X-ray diffraction pattern will not provide any visual cues of crystal twinning. For protein molecules, merohedrally twinned crystals exist predominately as hemihedrally twinned crystals (Yeates, 1997) which contain two distinct twin domains that are related to each other by a
Received 15 August 2009

Accepted 16 November 2009

PDB References: Nef $f_{\text {Core }}-$ TCR $\zeta_{\text {A63-R80 }}, 3$ ik5; $\mathrm{Nef}_{\text {core }}-\mathrm{TCR} \zeta_{\mathrm{DP} 1}, 3 \mathrm{ioz}$. 
twin-law operation. The twin fraction $\alpha$ represents the fractional contribution of the less prevalent twin domain. The diffraction pattern of a hemihedrally twinned crystal is therefore the superimposition of two unique diffraction patterns, one from each twin domain, where each reflection intensity is the weighted sum of two twin-related intensities (Grainger, 1969),

$$
\begin{aligned}
& I_{\mathrm{obs}}\left(h_{1}\right)=(1-\alpha) I\left(h_{1}\right)+\alpha I\left(h_{2}\right) \\
& I_{\mathrm{obs}}\left(h_{2}\right)=\alpha I\left(h_{1}\right)+(1-\alpha) I\left(h_{2}\right) .
\end{aligned}
$$

The individual intensities $I\left(h_{1}\right)$ and $I\left(h_{2}\right)$ can be solved by combining the linear equations

$$
\begin{aligned}
& I\left(h_{1}\right)=\frac{(1-\alpha) I_{\mathrm{obs}}\left(h_{1}\right)-\alpha I_{\mathrm{obs}}\left(h_{2}\right)}{1-2 \alpha} \\
& I\left(h_{2}\right)=\frac{-\alpha I_{\mathrm{obs}}\left(h_{1}\right)+(1-\alpha) I_{\mathrm{obs}}\left(h_{2}\right)}{1-2 \alpha} .
\end{aligned}
$$

As the twin fraction $\alpha$ approaches $1 / 2$ the crystal is considered to be perfectly twinned and calculation of the intensities $I\left(h_{1}\right)$ and $I\left(h_{2}\right)$ begins to fail as the term $(1-2 \alpha)$ begins to approach zero. This complicates the process of twin-related reflection intensity calculation, commonly referred to as detwinning. Structure determination has therefore preferentially been performed for hemihedral crystals that exhibit nonperfect twinning.

Less common cases of twinning have been described where a twin-law operation supports a higher Laue symmetry than that of the crystal unit cell (Rudolph et al., 2004). This type of twinning, which is referred to as pseudo-merohedral twinning, can occur in special circumstances such as a monoclinic system where the $\beta$ angle approaches $90^{\circ}$ (Larsen et al., 2002) or an orthorhombic system where the unit-cell axes $a$ and $b$ are fortuitously similar in length $(a \simeq b)$, resulting in the emulation of higher apparent tetragonal symmetry (Brooks et al., 2008). In this report, we describe such a case for crystals of a complex of the Nef (negative factor) protein from simian immunodeficiency virus bound to a fragment of one of Nef's cellular targets, the cytosolic domain of the TCR $\zeta$ subunit (TCR $\zeta$ ).

Nef from human immunodeficiency virus (HIV) or simian immunodeficiency virus (SIV) is a $27-35 \mathrm{kDa}$ viral accessory protein that is dispensable for replication but required for high infectivity and virulence (reviewed in Arien \& Verhasselt, 2008). Expressed in abundance early in the viral life cycle, Nef performs a number of functions that can be generalized into three activities: enhancement of viral infectivity, downregulation of surface receptors and modulation of T-cell activation. Notable among Nef's functions is the interaction of Nef with TCR $\zeta$ (Sigalov et al., 2008; Fackler et al., 2001; Swigut et al., 2000; Schaefer et al., 2000; Xu et al., 1999; Howe et al., 1998; Bell et al., 1998), the principal signaling component of the T-cell antigen receptor. This interaction has been suggested to play a role in HIV-mediated modulation of membraneproximal T-cell signaling events (Fenard et al., 2005; Thoulouze et al., 2006) and in SIV-mediated downregulation of the T-cell receptor (Schindler et al., 2006; Swigut et al., 2003;
Schaefer et al., 2002; Munch et al., 2002; Willard-Gallo et al., 2001). In previous work (Schaefer et al., 2000), SIV and HIV-2 Nef have been shown to bind $\operatorname{TCR} \zeta$ at two unique sites denoted 'SIV Nef interaction domains' (SNIDs), the first containing elements of immunoreceptor tyrosine activation motif (ITAM) 1 and the second containing elements of ITAM 2. However, the structural features of Nef that determine its specificity for TCR $\zeta$ remain unknown.

Nef contains two domains: an unstructured highly variable myristylated N-terminal domain and a C-terminal structured core domain (Arold et al., 1997; Grzesiek et al., 1997; Lee et al., 1996) that exhibits high sequence conservation among different HIV-1, HIV-2 and SIV isolates. The Nef conserved core domain $\left(\mathrm{Nef}_{\text {core }}\right)$ has been described to be responsible for the majority of Nef's interactions (Peter, 1998), including the TCR $\zeta$-binding activity of SIV Nef. The core domain of HIV-1 Nef has been shown to be amenable to crystallization (Arold et al., 1997; Lee et al., 1996; Franken et al., 1997). In this study, we aimed to determine the crystal structure of the Nef-TCR $\zeta$ complex by crystallizing complexes of SIVmac239 $\mathrm{Nef}_{\text {core }}$ with $\mathrm{TCR} \zeta$ fragment polypeptides containing the putative binding regions.

Here, we describe the crystallization and structure determination of the complexes of SIVmac239 $\mathrm{Nef}_{\text {core }}$ with two different $\mathrm{TCR} \zeta$ polypeptides, $\mathrm{TCR} \zeta_{\mathrm{DP} 1}$ and $\mathrm{TCR} \zeta_{\mathrm{A} 63-\mathrm{R} 80}$. Structure determination of the SIVmac239 $\mathrm{Nef}_{\text {core }}-\mathrm{TCR} \zeta_{\mathrm{DP} 1}$ complex was hampered by the poor electron-density maps calculated from the low-resolution diffraction data and phases derived from molecular replacement using the published HIV Nef core-domain structures. Eventually, we were able to determine this structure using the higher resolution SIVmac239 $\mathrm{Nef}_{\text {core }}-\mathrm{TCR} \zeta_{\mathrm{A} 63-\mathrm{R} 80}$ complex as a starting model. However, determination of the high-resolution SIVmac239 $\mathrm{Nef}_{\text {core }}-\mathrm{TCR} \zeta_{\mathrm{A} 63-\mathrm{R} 80}$ complex structure was hindered by the nearly perfect pseudo-merohedral crystal twinning that was detected on analysis of the intensity statistics. Ultimately, a partially twinned crystal with a twin fraction of 0.426 was used to solve the structure of the SIVmac239 $\mathrm{Nef}_{\text {core }}-\mathrm{TCR} \zeta_{\mathrm{A} 63-\mathrm{R} 80}$ complex to $2.05 \AA$ resolution. The structures of the two complexes revealed that crystallization of SIVmac239 $\mathrm{Nef}_{\text {core }}$ with the shorter $\mathrm{TCR} \zeta$ polypeptide had reduced the spacegroup symmetry from tetragonal to orthorhombic and introduced noncrystallographic symmetry (NCS). Because the unit-cell axes $a$ and $b$ were still nearly identical in the orthorhombic crystal form, the crystal was prone to twinning. This study presents a unique case in which pseudo-merohedral crystal twinning is the consequence of a reduction in crystal symmetry induced by the truncation of a protein ligand.

\section{Materials and methods}

\subsection{Protein expression and purification}

The core domain of SIVmac239 Nef with a two-residue linker, GS-Nef (Asp95-Ser235) ( Nef $\left._{\text {core }}\right)$, was expressed and purified as described by Sigalov et al. (2008). Briefly, Nef was expressed as a $6 \times$ His-thioredoxin fusion protein in Escher- 
ichia coli BL21 (DE3) cells. Following cell lysis, the Nef fusion protein was isolated by Ni-NTA affinity chromatography (Qiagen) under denaturing conditions (8M urea) and then dialyzed against a nondenaturing buffer containing $20 \mathrm{~m} M$ Tris, $150 \mathrm{mM} \mathrm{NaCl}, 100 \mu M$ DTT at $\mathrm{pH}$ 8.0. The soluble fusion protein was then subjected to proteolysis with thrombin (MP Biochemicals), resulting in the cleaved $\mathrm{Nef}_{\text {core }}$ protein. SIVmac239 $\mathrm{Nef}_{\text {core }}$ was purified by anion-exchange and sizeexclusion chromatography and concentrated to $700 \mu M$ by ultrafiltration (Amicon) in PBS.

TCR $\zeta_{\text {cyt }}$ includes an acid-labile Asp-Pro sequence (Landon, 1977) at positions 93-94. We made use of this to prepare two fragments of TCR $\zeta_{\text {cyt }}$, termed DP1 (Leu51-Asp93) and DP2 (Pro94-Arg164). TCR $\zeta_{\text {cyt }}$, purified as described by Sigalov et al. (2004), was incubated at $1.3 \mathrm{mg} \mathrm{ml}^{-1}(0.1 \mathrm{mM})$ in $30 \%$ acetonitrile, $0.5 \%(v / v)$ TFA for $48 \mathrm{~h}$ at $323 \mathrm{~K}$. Fragments were isolated by reverse-phase chromatography on a Vydac $\mathrm{C} 18$ $300 \AA$ pore-size column using an acetonitrile gradient in $0.1 \%$ TFA and recovered by lyophilization. Mass spectrometry was used to verify the identity of the fragments and the lack of any additional chemical modification other than the desired amide hydrolysis. All TCR $\zeta$ polypeptides were solubilized in $20 \mathrm{mM}$ Tris pH 8.0 to a final concentration of $700 \mu M$. Full-length $\mathrm{TCR} \zeta$ was expressed and purified as reported previously (Sigalov et al., 2004).

\subsection{Crystallization}

Crystals of SIVmac239 $\mathrm{Nef}_{\text {core }}$ in complex with the TCR $\zeta$ polypeptides TCR $\zeta_{\mathrm{DP} 1}$ and TCR $\zeta_{\mathrm{A} 63-\mathrm{R} 80}$ were grown at $277 \mathrm{~K}$ using the hanging-drop vapor-diffusion method (McPherson, 1982). Crystals of the $\mathrm{Nef}_{\text {core }}-\mathrm{TCR} \zeta_{\mathrm{DP} 1}$ complex were grown in a $2 \mu \mathrm{l}$ hanging drop by mixing $0.5 \mu \mathrm{l}$ SIVmac239 $\mathrm{Nef}_{\text {core }}$ $(700 \mu M), 0.5 \mu \mathrm{TCR} \zeta_{\mathrm{DP} 1}(700 \mu M)$ and $1 \mu \mathrm{l}$ crystallization buffer (15\% PEG 3350, $150 \mathrm{~m} M$ KF, $100 \mathrm{~m} M$ HEPES pH 8.2). Crystals of the $\mathrm{Nef}_{\text {core }}-\mathrm{TCR} \zeta_{\mathrm{A} 63-\mathrm{R} 80}$ complex were grown in a $3 \mu \mathrm{l}$ hanging drop by mixing $1 \mu \mathrm{l} \mathrm{SIVmac239} \mathrm{Nef}_{\text {core }}(700 \mu M)$, $1 \mu \mathrm{l} \mathrm{TCR} \zeta_{\mathrm{A} 63-\mathrm{R} 80}(700 \mu M)$ and $1 \mu \mathrm{l}$ crystallization buffer $(10-$ $15 \%$ PEG 3350, $200 \mathrm{~m} M \mathrm{NH}_{4} \mathrm{~F}, 100 \mathrm{~m} M$ HEPES $\mathrm{pH}$ 7.4-7.5). The hanging drops were suspended on siliconized glass cover slips over $1 \mathrm{ml}$ crystallization buffer in 24-well plates (Vydax). Crystals of the $\mathrm{Nef}_{\text {core }}-\mathrm{TCR} \zeta$ polypeptide complexes grew to maximal size $(750 \times 150 \times 150 \mu \mathrm{m})$ in 3-7 d. Prior to X-ray diffraction experiments, the $\mathrm{Nef}_{\text {core }}-\mathrm{TCR} \zeta_{\mathrm{DP} 1}$ and $\mathrm{Nef}_{\text {core }}{ }^{-}$ $\mathrm{TCR} \zeta_{\mathrm{A} 63-\mathrm{R} 80}$ crystals were transferred into cryoprotectant solutions containing $20-25 \%$ ethylene glycol in their respective crystallization buffers and then flash-cooled in liquid nitrogen.

\subsection{Data collection and processing}

16 data sets were collected from various $\mathrm{Nef}_{\text {core }}-\mathrm{TCR} \zeta$ crystals, of which three were used for structure determination. One low-resolution data set $(3.7 \AA)$ for the SIVmac239 $\mathrm{Nef}_{\text {core }}-\mathrm{TCR} \zeta_{\mathrm{DP} 1}$ complex and two high-resolution data sets $(1.9$ and $2.05 \AA)$ for the SIVmac239 $\mathrm{Nef}_{\text {core }}-\mathrm{TCR} \zeta_{\mathrm{A} 63-\mathrm{R} 80}$ complex were collected from single crystals at the National Synchrotron Light Source (beamline X29) using an ADSC
Table 1

Data-collection and refinement statistics (molecular replacement).

Values in parentheses are for the highest resolution shell.

\begin{tabular}{|c|c|c|c|}
\hline & $\begin{array}{l}\text { Nef }_{\text {core }}- \\
\text { TCR } \zeta_{\text {DP1 }}\end{array}$ & $\begin{array}{l}\text { Nef }_{\text {core }}- \\
\text { TCR } \zeta_{\text {A63-R80 }}, \\
\text { crystal } 1\end{array}$ & $\begin{array}{l}\mathrm{Nef}_{\text {core }}{ }^{-} \\
\text {TCR } \zeta_{\text {A63-R } 80}, \\
\text { crystal 2 }\end{array}$ \\
\hline \multicolumn{4}{|l|}{ Data collection } \\
\hline Space group & $P 4_{3} 2_{1} 2$ & $P 2_{1} 2_{1} 2_{1}$ & $P 2_{1} 2_{1} 2_{1}$ \\
\hline \multicolumn{4}{|l|}{ Unit-cell parameters } \\
\hline $\begin{array}{l}a(\AA) \\
b(\AA)\end{array}$ & $\begin{array}{l}51.64 \\
51.64\end{array}$ & $\begin{array}{l}47.19 \\
47.24\end{array}$ & $\begin{array}{l}47.42 \\
47.42\end{array}$ \\
\hline$c(\AA)$ & 189.45 & 182.99 & 183.52 \\
\hline$\alpha=\beta=\gamma\left({ }^{\circ}\right)$ & 90 & 90 & 90 \\
\hline Resolution $(\AA \stackrel{\AA}{)}$ & $\begin{array}{l}50-3.70 \\
\quad(3.83-3.70)\end{array}$ & $\begin{array}{l}50-1.93 \\
\quad(2.02-1.93)\end{array}$ & $\begin{array}{l}30-2.05 \\
\quad(2.12-2.05)\end{array}$ \\
\hline$R_{\text {merge }}$ & $0.051(0.393)$ & $0.083(0.513)$ & $0.084(0.498)$ \\
\hline$I / \sigma(I)$ & $12.8(2.1)$ & $9.7(2.4)$ & $9.6(2.5)$ \\
\hline Completeness (\%) & $99.4(100.0)$ & $99.5(98.8)$ & $99.1(96.5)$ \\
\hline Redundancy & $12.6(12.7)$ & $6.9(5.9)$ & $6.8(5.2)$ \\
\hline Twin fraction $(k, h,-l)$ & N/A & 0.500 & 0.426 \\
\hline \multicolumn{4}{|l|}{ Refinement } \\
\hline Resolution $(\AA)$ & $36-3.70$ & & $27-2.05$ \\
\hline Total reflections & 2923 & & 25896 \\
\hline \multirow{2}{*}{\multicolumn{4}{|c|}{$\begin{array}{l}N_{\text {work }} / I_{\text {free }} \\
\text { No. of atoms }\end{array}$}} \\
\hline & & & \\
\hline Protein & 1054 & & 2223 \\
\hline Water & N/A & & 116 \\
\hline \multirow{2}{*}{\multicolumn{4}{|c|}{ Average $B$ factors $\left(\AA^{2}\right)$}} \\
\hline & & & \\
\hline SIVmac239 Nef $_{\text {core }}$ & 204.63 & & 46.0 \\
\hline $\mathrm{TCR} \zeta$ polypeptide & 219.51 & & 49.2 \\
\hline Waters & N/A & & 45.2 \\
\hline \multicolumn{4}{|l|}{ R.m.s. deviations } \\
\hline Bond lengths (£) & 0.003 & & 0.003 \\
\hline Bond angles $\left({ }^{\circ}\right)$ & 0.565 & & 0.564 \\
\hline PDB code & $3 \mathrm{ioz}$ & & $3 i k 5$ \\
\hline
\end{tabular}

Quantum-315r CCD detector system. The crystal-to-detector distances for the $\mathrm{Nef}_{\text {core }}-\mathrm{TCR} \zeta_{\mathrm{DP} 1}$ complex and the $\mathrm{Nef}_{\text {core }}{ }^{-}$ $\mathrm{TCR} \zeta_{\mathrm{A} 63-\mathrm{R} 80}$ complex crystals were 275 and $250 \mathrm{~mm}$, respectively. The crystals were exposed for $1 \mathrm{~s}$ with an oscillation of $1^{\circ}$ per image. A total of 180 images were collected for each data set, which were separately indexed, integrated and scaled in HKL-2000 (Otwinowski \& Minor, 1997). Detection and analysis of crystal twinning was performed in phenix.xtriage from the PHENIX software package (Adams et al., 2002). Determination of the twin law governing the pseudo-merohedrally twinned SIVmac239 $\mathrm{Nef}_{\text {core }}-\mathrm{TCR} \zeta_{\text {A63-R80 }}$ crystals was performed following proper assignment of the crystal space group as described below.

\subsection{Structure determination and refinement}

The structures of the SIVmac239 $\mathrm{Nef}_{\text {core }}-\mathrm{TCR} \zeta_{\mathrm{A63}-\mathrm{R} 80}$ and SIVmac239 $\mathrm{Nef}_{\text {core }}-\mathrm{TCR} \zeta_{\mathrm{DP} 1}$ complexes were determined by molecular replacement. Firstly, the atomic coordinates of the Nef core domain from HIV-1 were extracted from the crystal structures of the unliganded HIV-1 isolate LAI Nef structure (PDB code 1avv; Arold et al., 1997), the HIV-1 isolate LAI Nef-Fyn SH3 domain complex (PDB code 1avz; Arold et al., 1997) and HIV-1 isolate NL4-3 Nef-Fyn SH3 (R96I) domain complex (PDB code 1efn; Lee et al., 1996) and were modified with CHAINSAW (Stein, 2008) to trim the side chains not shared by SIVmac239 Nef (44\% sequence identity) to methyl 
groups. The modified Nef coordinate sets were used as an ensemble search model for molecular replacement in Phaser (Storoni et al., 2004) and single solutions with translationfunction $Z$ scores (TFZ) greater than 6.0 were used as starting models. The structure of the $\mathrm{Nef}_{\text {core }}-\mathrm{TCR} \zeta_{\mathrm{A} 63-\mathrm{R} 80}$ complex was solved by multiple rounds of twinned refinement using phenix.refine from the PHENIX software package (Adams et al., 2002) interspersed with rounds of manual model building and fitting of $F_{\mathrm{o}}-F_{\mathrm{c}}$ and $2 F_{\mathrm{o}}-F_{\mathrm{c}}$ electron-density maps in Coot (Emsley \& Cowtan, 2004). The twin operator $(k, h,-l)$ was applied during each round of refinement, which included three cycles of individual atomic displacement factor refinement and individual energy-minimization procedures accompanied by refinement of the twin fraction $\alpha$. Water molecules were added to the refined model using both phenix.refine and Coot. The quality of the final refined SIVmac239 $\mathrm{Nef}_{\text {core }}-\mathrm{TCR} \zeta_{\text {A63-R80 }}$ structure was validated in PROCHECK (Laskowski et al., 1993).

The structure of the refined SIVmac239 $\mathrm{Nef}_{\text {core }}{ }^{-}$ $\mathrm{TCR} \zeta_{\mathrm{A} 63-\mathrm{R} 80}$ complex was used as a search model to find a molecular-replacement solution for the SIVmac239 $\mathrm{Nef}_{\text {core }}{ }^{-}$ $\mathrm{TCR} \zeta_{\mathrm{DP} 1}$ data. A single top molecular-replacement solution $(\mathrm{TFZ}=10.5, \mathrm{LLG}=266)$ was found and used as a starting model. The structure of the SIVmac239 $\mathrm{Nef}_{\text {core }}-\mathrm{TCR} \zeta_{\text {DP1 }}$ structure was solved by refinement consisting of several rounds of individual atomic displacement factor refinement and individual energy-minimization procedures using phenix.refine. Model inspection was performed between each round of refinement and the model was modified in Coot. The final refined structure was validated for acceptable chemical properties with PROCHECK. Final model and refinement statistics for both SIVmac239 $\mathrm{Nef}_{\text {core }}-\mathrm{TCR} \zeta$ polypeptide structures are shown in Table 1 and Ramachandran plots generated by RAMPAGE (Lovell et al., 2003) are provided in Supplementary Figs. S1 and S2 ${ }^{\mathbf{1}}$.

\section{Results and discussion}

\subsection{Crystallization and data collection of two SIVmac239 $\mathrm{Nef}_{\text {core }}-\mathrm{TCR} \zeta$ polypeptide complexes}

In order to determine the structure of the Nef-TCR $\zeta$ complex, mixtures of the structured core domain of SIVmac239 Nef (Asp95-Ser235) with various polypeptides spanning the putative binding regions of TCR $\zeta$ (Schaefer $e t$ al., 2000) were screened for crystal formation. Initial crystallization experiments were aimed towards crystallizing the complex of $\mathrm{Nef}_{\text {core }}$ (SIVmac239, HIV-1 ELI and NL4-3) with the full-length cytoplasmic domain of $\mathrm{TCR} \zeta\left(\mathrm{TCR} \zeta_{\text {cyt }}\right)$, but these were unsuccessful. Therefore, a polypeptide crystallization screening strategy was employed to identify a minimal TCR $\zeta$ polypeptide that bound SIVmac239 $\mathrm{Nef}_{\text {core }}$, which exhibited the highest affinity TCR $\zeta_{\text {cyt }}$ binding among the SIV,

\footnotetext{
${ }^{\mathbf{1}}$ Supplementary material has been deposited in the IUCr electronic archive (Reference: YT5020). Services for accessing this material are described at the back of the journal.
}

HIV-1 and HIV-2 variants tested (SIVmac239, HIV-1 ELI and NL4-3, and HIV-2 ST; unpublished results).

Crystallization efforts focused on TCR $\zeta$ fragments that contained the N-terminal of the two SIV-interaction domains (Fig. 1). Of a series of polypeptides spanning $\mathrm{TCR} \zeta_{\mathrm{cyt}}$, a peptide included in this region, $\mathrm{TCR} \zeta_{\mathrm{A} 61-\mathrm{R} 80}$, bound to $\mathrm{Nef}_{\text {core }}$ from HIV-1, HIV-2 and SIV strains (manuscript in preparation) and the structural information obtained for the higher affinity SIV variant might be relevant for HIV-1 as well as the more homologous HIV-2 Nef proteins. Moreover, TCR $\zeta_{\mathrm{DP} 1}$ (i.e. the $\mathrm{N}$-terminal acid-cleavage fragment, residues Leu51Asp93) formed a 1:1 stoichiometric complex with SIVmac239 $\mathrm{Nef}_{\text {core }}$ (unpublished results), as did intact TCR $\zeta_{\text {cyt }}$ (Sigalov et al., 2008). Therefore, a series of polypeptides containing the original $\mathrm{TCR} \zeta_{\mathrm{DP} 1}$ polypeptide, the shorter $\mathrm{TCR} \zeta_{\mathrm{A} 61-\mathrm{R} 80}$ polypeptide and several peptides containing the proposed SNID-1 (Schaefer et al., 2000) sequence were either prepared from full-length $\mathrm{TCR} \zeta_{\mathrm{cyt}}\left(\mathrm{TCR} \zeta_{\mathrm{DP} 1}\right)$ or chemically synthesized (TCR $\zeta_{\mathrm{A} 61-\mathrm{R} 80}$ and variants) and used in crystallization experiments with SIVmac239 $\mathrm{Nef}_{\text {core }}$.

SIVmac239 Nef $_{\text {core }}$ crystallized in complex with TCR $\zeta_{\text {DP1 }}$ and $\mathrm{TCR} \zeta_{\mathrm{A} 63-\mathrm{R} 80}$ under similar conditions. Crystals of the SIVmac239 $\mathrm{Nef}_{\text {core }}-\mathrm{TCR} \zeta_{\mathrm{DP} 1}$ complex grew readily as long tetragonal pyramids (Fig. $2 a$ ) but diffracted X-rays to low resolution ( $3.7 \AA$ A Fig. $2 b$ ) and could not be improved further by optimization of the crystallization conditions. In contrast, crystals of SIVmac239 $\mathrm{Nef}_{\text {core }}$ bound to TCR $\zeta_{\mathrm{A63}-\mathrm{R} 80}$ adopted a bipyramidal shape (Fig. 2a) and diffracted X-rays to high resolution (1.9-2.05 $\AA$; Fig. $2 b$ ). Neither crystal form exhibited the concave or 're-entrant' features that have been suggested to predict the presence of twinned crystals (Yeates, 1997), nor did their diffraction patterns contain split reflections (Fig. 2c).

\subsection{Space-group determination and molecular replacement for SIVmac239 $\mathrm{Nef}_{\text {core }}-\mathrm{TCR} \zeta_{\mathrm{DP} 1}$}

The low-resolution diffraction data for the SIVmac239 $\mathrm{Nef}_{\text {core }}-\mathrm{TCR} \zeta_{\text {DP1 }}$ complex were indexed in the tetragonal Laue group 4/mmm (422 point group), with unit-cell parameters $a=b=51.638, c=189.449 \AA$ and an $R_{\text {merge }}$ of $5.1 \%$. The Matthews coefficient $V_{\mathrm{M}}$ (Matthews, 1968) was calculated to be $2.86 \AA^{3} \mathrm{Da}^{-1}$ (57.1\% solvent), indicating the presence of one SIVmac239 $\mathrm{Nef}_{\text {core }}-\mathrm{TCR} \zeta_{\mathrm{DP} 1}$ heterodimer per asym-

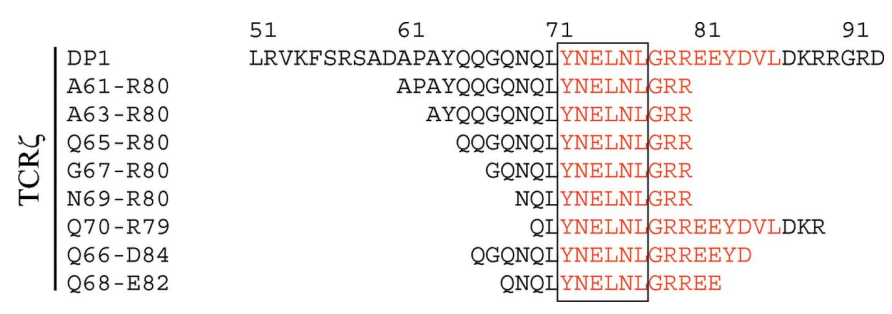

Figure 1

$\mathrm{TCR} \zeta$ polypeptide crystallization screen. The polypeptide sequences are shown with residue position numbers assigned on the left. The boxed region contains the sequence of the first of the two reported SIV Nef interaction domains (Schaefer et al., 2000). The sequence of ITAM 1 is colored red. 
metric unit. After analysis of the $h 00,0 k 0$ and $00 l$ reflection intensities, the space group was further assigned as $P 4_{3} 2_{1} 2$ or $P 4_{1} 2_{1} 2$ based on the indicated presence of screw axes along $a$ and $c$. Using an ensemble of HIV-1 Nef core domain structures as a search model, a single molecular-replacement solution $(\mathrm{TFZ}=9.4, \mathrm{LLG}=63)$ was found in space group $P 4_{3} 2_{1} 2$. However, model refinement and building were hindered by the poor quality of the $\sigma_{\mathrm{A}}$-weighted $F_{\mathrm{o}}-F_{\mathrm{c}}$ and $2 F_{\mathrm{o}}-F_{\mathrm{c}}$ electron-density maps, resulting in an $R_{\text {free }}$ that could not be reduced below $41 \%$.

\subsection{Initial space-group determination and molecular replacement for \\ SIVmac239 Nef $_{\text {core }}-$ TCR $\zeta_{\text {A63-R80 }}$}

Two crystals of SIVmac239 $\mathrm{Nef}_{\text {core }}$ bound to the shorter $\mathrm{TCR} \zeta_{\mathrm{A} 63-\mathrm{R} 80}$ polypeptide diffracted $\mathrm{X}$-rays to higher resolution (1.9-2.05 $⿱$ ) , but spacegroup determination proved to be more complicated than for the $P 4_{3} 2_{1} 2$ crystal form of $\mathrm{Nef}_{\text {core }}-\mathrm{TCR} \zeta_{\mathrm{DP} 1}$ described above. The diffraction patterns that were observed for $\mathrm{Nef}_{\text {core }}-\mathrm{TCR} \zeta_{\mathrm{A63}-\mathrm{R} 80}$ appeared to be consistent with the lattice previously observed for the lower resolution $\mathrm{Nef}_{\text {core }}-\mathrm{TCR} \zeta_{\mathrm{DP} 1}$ crystals, and the TCR $\zeta_{\mathrm{A} 63-\mathrm{R} 80}$ data (crystal 1) were initially indexed in the same tetragonal Laue group 4/mmm (422 point group), with unit-cell parameters $a=b=47.203, c=182.939 \AA$ ․ The integration statistics were similar to those observed previously $\left(R_{\text {merge }}=\right.$ $7.6 \%)$. Visual inspection of reflection intensities using HKLVIEW (Collaborative Computational Project, Number 4,1994$)$ and evalution of the $R_{\text {merge }}$ and $\Delta I / \sigma(I)$ statistics confirmed the presence of each of the symmetry elements comprising the 422 point group. All of the unit-cell parameters were reduced by $4-10 \%$ compared with the SIVmac239 $\mathrm{Nef}_{\text {core }}-\mathrm{TCR} \zeta_{\mathrm{DP} 1}$ crystals, which is potentially consistent with the shorter length of the TCR $\zeta$ polypeptide ligand. Analysis of the $h 00$ and $0 k 0$ intensities indicated the presence of $2_{1}$ screw axes along $a$ and $b$. However, $00 l$ intensities were observed for $l=2 n$, which is consistent with a $4_{2}$ (or $2_{1}$ ) axis along $c$ but inconsistent with a $4_{3}$ axis as observed for the lower resolution tetragonal SIVmac239 $\mathrm{Nef}_{\text {core }}-\mathrm{TCR} \zeta_{\mathrm{DP} 1}$ data. A search for a molecular-replacement solution for the SIVmac239

\section{Figure 2}

$\mathrm{Nef}_{\text {core }}-\mathrm{TCR} \zeta_{\mathrm{A} 63-\mathrm{R} 80}$ data in $4_{2} 2_{1} 2$ or any of the other 422 space groups yielded no solutions. A data set that was collected from a second SIVmac239 $\mathrm{Nef}_{\text {core }}-\mathrm{TCR} \zeta_{\mathrm{A} 63-\mathrm{R} 80}$ crystal (crystal 2) resulted in similar difficulties with molecular replacement. The ambiguous space-group assignment and the inability to find a molecular-replacement solution for the SIVmac239 $\mathrm{Nef}_{\text {core }}-\mathrm{TCR} \zeta_{\mathrm{A} 63-\mathrm{R} 80}$ complex in the tetragonal
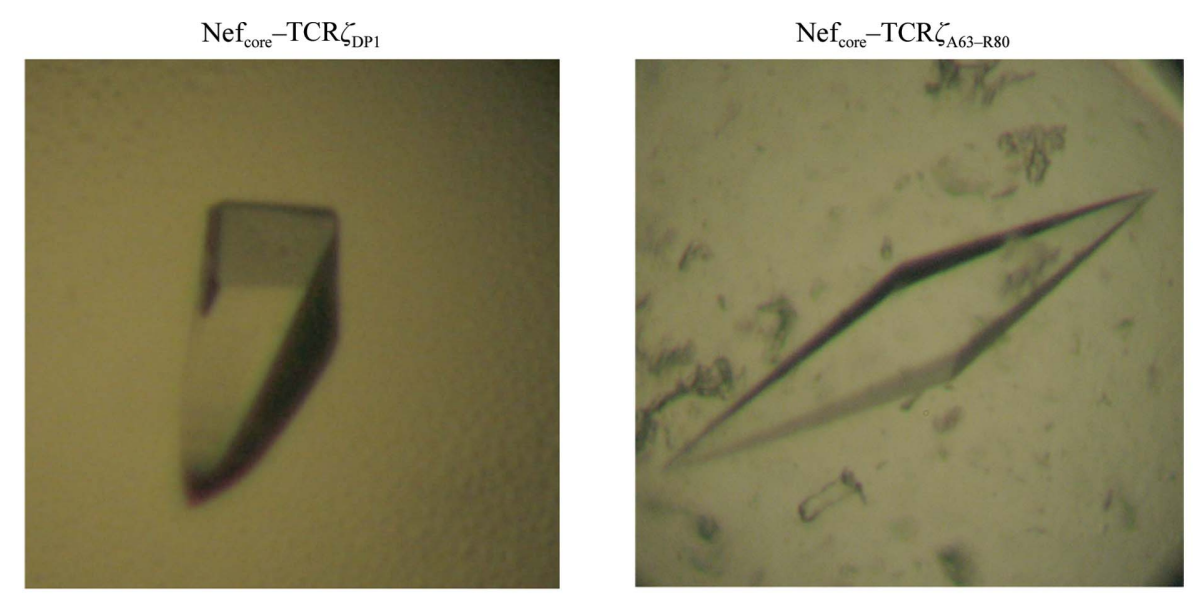

(a)
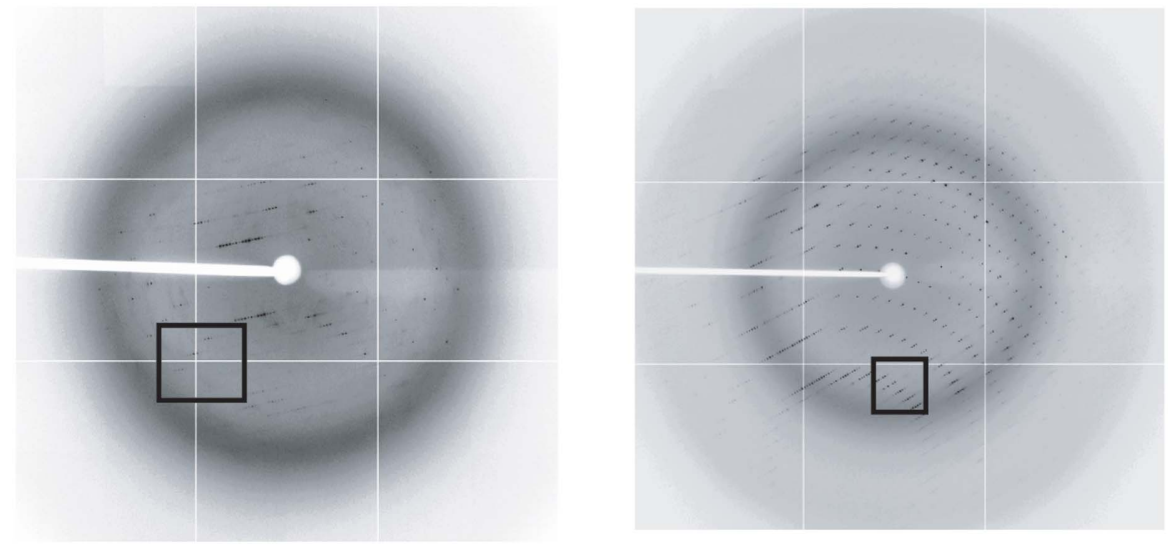

(b)
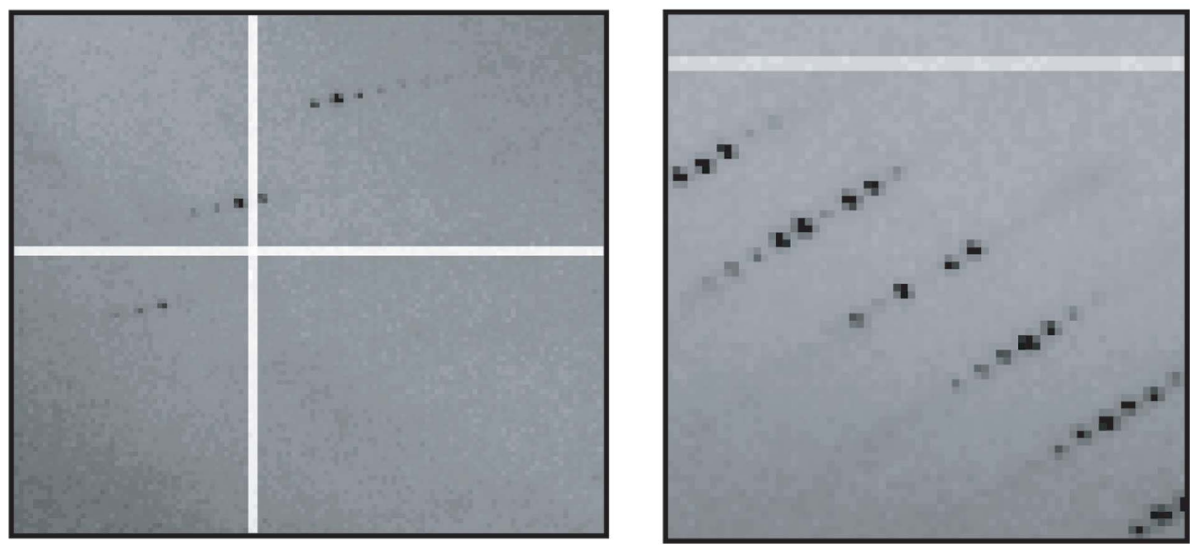

(c)

Crystallization and diffraction. (a) Crystals of the SIVmac239 $\mathrm{Nef}_{\text {core }}-\mathrm{TCR} \zeta_{\mathrm{DP} 1}$ and SIVmac239 $\mathrm{Nef}_{\text {core }}-\mathrm{TCR} \zeta_{\mathrm{A} 63-\mathrm{R} 80}$ complexes. Both crystals grew to $750 \times 150 \times 150 \mu \mathrm{m}$ at $277 \mathrm{~K}$. (b) Diffraction patterns of crystals of the SIVmac239 $\mathrm{Nef}_{\text {core }}-\mathrm{TCR} \zeta$ polypeptide complexes collected on beamline X29 at the National Light Synchrotron Light Source, Brookhaven National Laboratory. (c) Enlarged view of the diffraction patterns. The diffraction-pattern spot profiles are singular, with no evidence of split spots. 
Laue symmetry group suggested the possibility of crystal twinning and required space-group re-evaluation.

\subsection{Detection and analysis of twinning}

A number of statistical methods have been developed to characterize crystal twinning, including the recently developed Padilla-Yeates algorithm for detection of the presence of crystal twinning (Padilla \& Yeates, 2003) and the Britton plot for estimation of the twin fraction $\alpha$ (Britton, 1972). To assess the twinning of the SIVmac239 $\mathrm{Nef}_{\text {core }}-\mathrm{TCR} \zeta$ polypeptide crystals, several analyses of the intensity statistics were performed in phenix.xtriage. Firstly, the second moments of the intensities of acentric data $\left(\left\langle I^{2}\right\rangle /\left\langle|I|^{2}\right\rangle\right)$ were calculated for all three SIVmac239 $\mathrm{Nef}_{\text {core }}-\mathrm{TCR} \zeta$ polypeptide complexes. Untwinned and twinned data are expected to have $\left\langle I^{2}\right\rangle /\left\langle|I|^{2}\right\rangle$
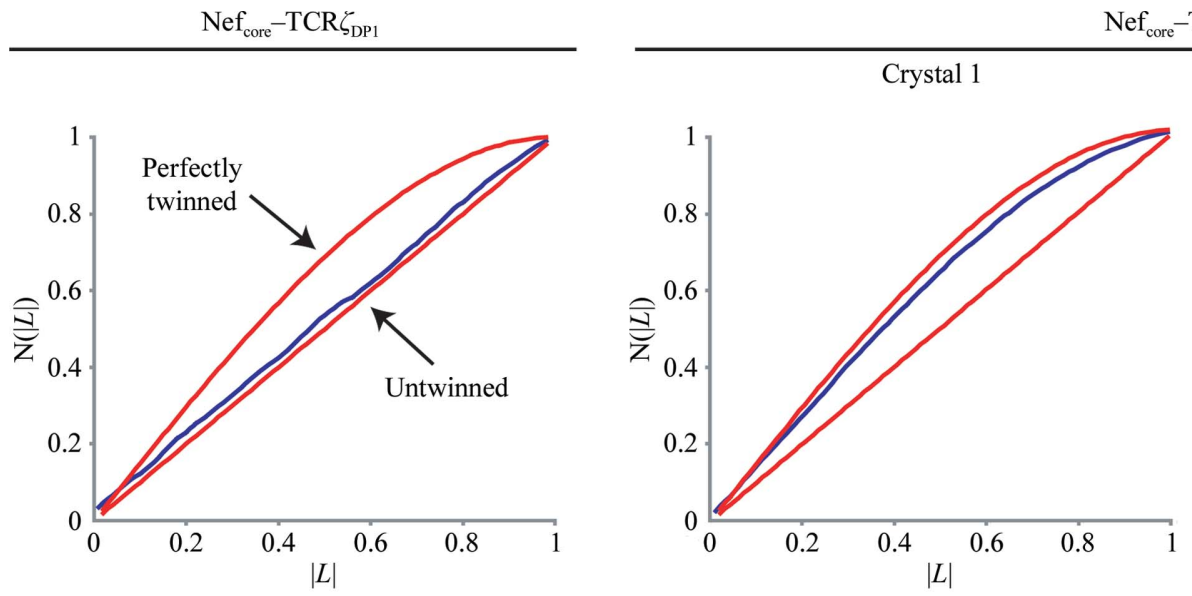

$\mathrm{Nef}_{\text {core }}-\mathrm{TCR} \zeta_{\mathrm{A} 63-\mathrm{R} 80}$
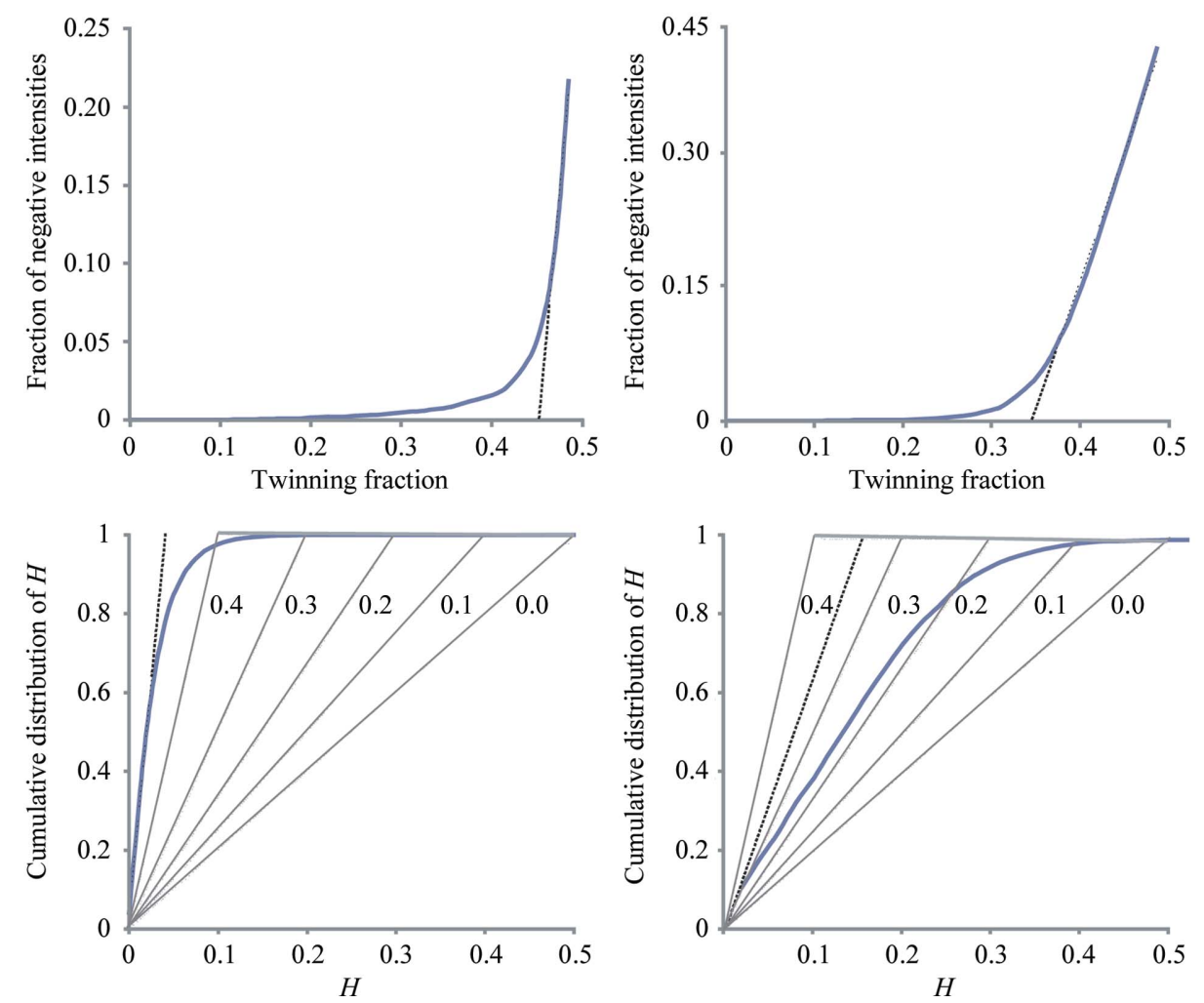

Figure 3

Detection of twinning and estimation of the twin fraction $\alpha$. Top row, cumulative intensity difference plot of the intensity difference of local pairs of intensities that are not twin-related $|L|\left\{L=\left[I\left(h_{1}\right)-I\left(h_{2}\right)\right] /\left[I\left(h_{1}\right)+I\left(h_{2}\right)\right]\right\}$ against the cumulative probability distribution $N(L)$ of the parameter $L$ (Padilla \& Yeates, 2003). The expected plots for untwinned and twinned acentric data (red) and the calculated plots for the SIVmac239 Nef core $^{-T C R} \zeta$ polypeptide data (blue) are shown. Middle row, estimation of the twin fraction $\alpha$ by Britton plot analysis (Britton, 1972). The percentage of negative intensities after detwinning is plotted as a function of the assumed value of $\alpha$. Overestimation of the twin factor $\alpha$ results in an increase in the percentage of negative intensities. The estimated value of $\alpha$ is extrapolated from the linear fit (dashed line). Bottom row, estimation of the twin fraction $\alpha$ using the $H$ plot (Yeates, 1988). The cumulative fractional intensity difference of acentric twin-related intensities $H\left\{H=\left|I\left(h_{1}\right)-I\left(h_{2}\right)\right| /\left[I\left(h_{1}\right)+I\left(h_{2}\right)\right]\right\}$ is plotted against $H$. The initial slope (dashed line) of the distribution is a measure of $\alpha$. The expected slopes for the indicated twin fractions $0.0-0.4$ are shown (dotted lines). 
values of 2.0 and 1.5 , respectively. The SIVmac239 $\mathrm{Nef}_{\text {core }}{ }^{-}$ $\mathrm{TCR} \zeta_{\mathrm{DP} 1}$ crystal had an $\left\langle I^{2}\right\rangle /\left\langle|I|^{2}\right\rangle$ value of 2.106 , suggesting

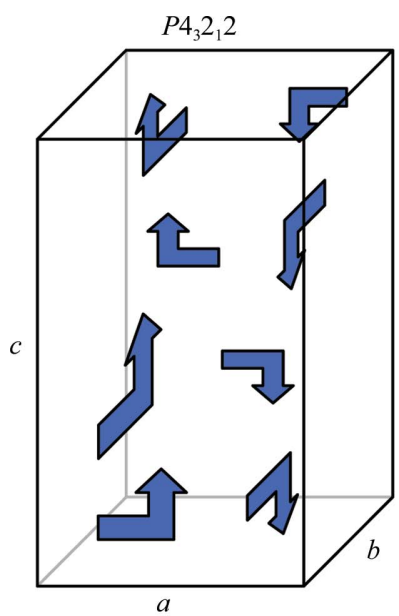

(a)

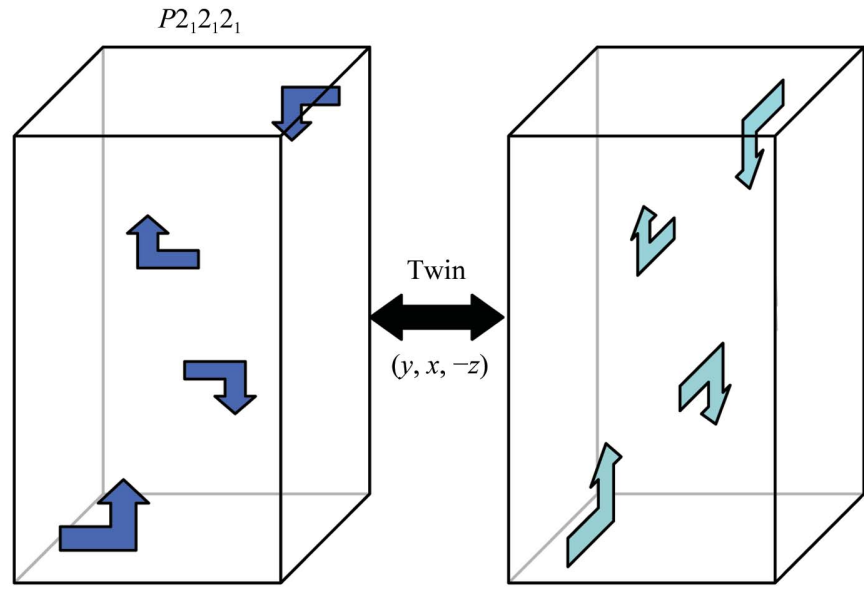

(b)

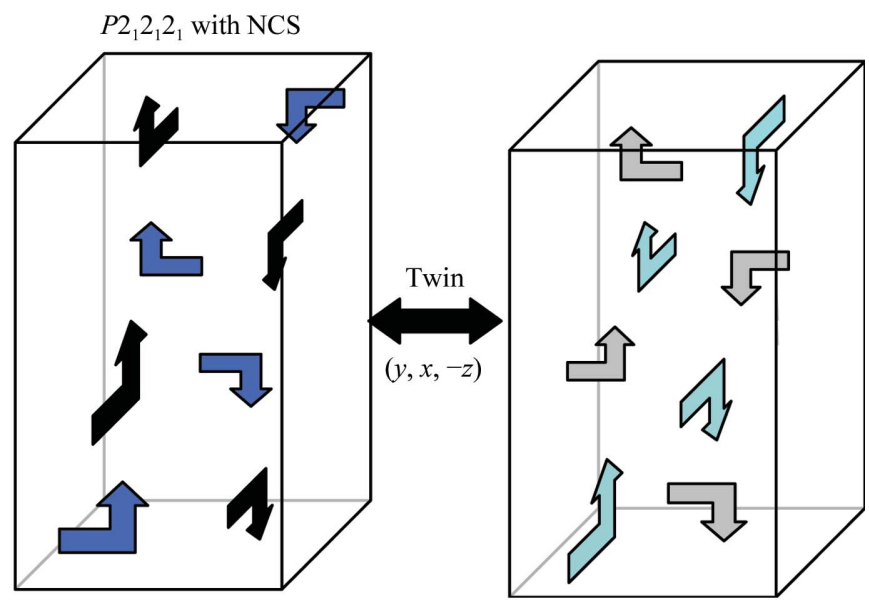

(c)

Figure 4

Twinning in an orthorhombic $P 2_{1} 2_{1} 2_{1}$ crystal. (a) A $P 4_{3} 2_{1} 2$ space-group unit cell with one molecule (arrow) per ASU (eight per unit cell) is shown with axes $a, b$ and $c$ labeled. (b) A $P 2_{1} 2_{1} 2_{1}$ space-group unit cell with one molecule per ASU (four per unit cell) is shown (left) with its twin unit cell (right) related by the twin operator $(y, x,-z)$. (c) A $P 2_{1} 2_{1} 2_{1}$ space-group unit cell with two molecules per ASU (four per unit cell) is shown (left) with its twin unit cell (right) related by the twin operator $(y, x,-z)$. The ASU is comprised of one blue and one black arrow related by noncrystallographic symmetry. the absence of twinning, whereas the SIVmac239 $\mathrm{Nef}_{\text {core }}{ }^{-}$ $\mathrm{TCR} \zeta_{\mathrm{A} 63-\mathrm{R} 80}$ crystals had $\left\langle I^{2}\right\rangle /\left\langle|I|^{2}\right\rangle$ values of 1.676 (crystal 1) and 1.628 (crystal 2), indicating the presence of twinning in both crystals. A more robust method of twin detection that uses cumulative local intensity deviation distribution statistics as determined by the Padilla-Yeates algorithm (Padilla \& Yeates, 2003) was also employed. In a plot of the local intensity difference $|L|$ of non-twin-related intensities versus the distribution of the local intensity differences $N|L|$, the presence of twinning can be deduced by comparing the experimental plots with the expected plots for twinned and untwinned data (Padilla \& Yeates, 2003). The SIVmac239 $\mathrm{Nef}_{\text {core }}-\mathrm{TCR} \zeta_{\mathrm{DP} 1}$ data plot was linear, which is consistent with the expected curve for untwinned data (Fig. 3, top). In contrast, the plots for both SIVmac239 $\mathrm{Nef}_{\text {core }}-\mathrm{TCR} \zeta_{\mathrm{A} 63-\mathrm{R} 80}$ crystals 1 and 2 were curved, suggesting the presence of crystal twinning (Fig. 3, top). The $L$ test, which is also based on the local intensity differences of non-twin-related reflection pairs, was additionally employed in order to confirm twinning in the SIVmac239 $\mathrm{Nef}_{\text {core }}-\mathrm{TCR} \zeta_{\mathrm{A} 63-\mathrm{R} 80}$ crystals; for untwinned data $|L|$ and mean $L^{2}$ are expected to be $1 / 2$ and $1 / 3$, respectively, and for twinned data they are expected to be $3 / 8$ and $1 / 5$, respectively. The SIVmac239 $\mathrm{Nef}_{\text {core }}-\mathrm{TCR} \zeta_{\mathrm{DP} 1}$ data had calculated $|L|$ and $L^{2}$ values of 0.473 and 0.307 , which were consistent with an absence of appreciable twinning. The SIVmac239 $\mathrm{Nef}_{\text {core }}-\mathrm{TCR} \zeta_{\mathrm{A} 63-\mathrm{R} 80}$ crystals had calculated $|L|$ and $L^{2}$ values of 0.402 and 0.229 for crystal 1 and 0.390 and 0.218 for crystal 2 , further supporting the presence of crystal twinning. All of the twinning tests suggested that the lowresolution SIVmac239 $\mathrm{Nef}_{\text {core }}-\mathrm{TCR} \zeta_{\text {DP1 }}$ crystal was not appreciably twinned, whereas the high-resolution SIVmac239 $\mathrm{Nef}_{\text {core }}-\mathrm{TCR} \zeta_{\mathrm{A63}-\mathrm{R} 80}$ crystals were pseudo-merohedrally twinned with a twin fraction near 0.5 .

In order to estimate the twin fraction $\alpha$ in the two pseudomerohedrally twinned SIVmac239 $\mathrm{Nef}_{\text {core }}-\mathrm{TCR} \zeta_{\mathrm{A} 63-\mathrm{R} 80}$ crystals, Britton plot (Britton, 1972) and $H$-plot (Yeates, 1988) analyses were performed (Fig. 3, middle and bottom). Crystal 1 exhibited near-perfect twinning, with an estimated twin fraction of 0.452 from the Britton plot and of 0.477 from the $H$ plot. In contrast, crystal 2 seemed to be only partially twinned, with estimated twin fractions of 0.344 and 0.356 from the Britton plot and $H$ plot, respectively. These initial estimates of the twin fraction based on statistical analysis of intensities were found to underestimate the actual twin fraction, which refined upwards during structure determination to 0.500 and 0.426 for crystals 1 and 2, respectively (see below).

Because the Laue group 4/ $\mathrm{mmm}$ does not support merohedral twinning, we explored the possibility that the twinned SIVmac239 $\mathrm{Nef}_{\text {core }}-\mathrm{TCR} \zeta_{\text {A63-R80 }}$ crystals were orthorhombic crystals that emulated tetragonal symmetry owing to pseudomerohedral crystal twinning. The twinned SIVmac239 $\mathrm{Nef}_{\text {core }}{ }^{-}$ $\mathrm{TCR} \zeta_{\mathrm{A63-R} 80}$ diffraction data were therefore re-indexed in the orthorhombic point group 222. The unit-cell parameters $a$ and $b$ that are constrained to be equal in point group 422 (Laue group 4/mmm) refined to slightly different values of $a=47.197$ and $b=47.208 \AA$ for crystal 1 and $a=47.417$ and $b=47.421 \AA$ for crystal 2, with no significant changes in $R_{\text {merge }}$ values 
(Table 1). Inspection of the $h 00,0 k 0$ and $00 l$ intensities indicated the presence of $2_{1}$ screw axes along each axis, suggesting a $P 2_{1} 2_{1} 2_{1}$ crystal space group. The Matthews coefficient $V_{\mathbf{M}}$ (Matthews, 1968) was calculated to be $2.64 \AA^{3} \mathrm{Da}^{-1}(53.42 \%$ solvent) for crystal 1 and $2.64 \AA^{3} \mathrm{Da}^{-1}$ (53.99\% solvent) for crystal 2, which is consistent with two SIVmac239 $\mathrm{Nef}_{\text {core }}{ }^{-}$ $\mathrm{TCR} \zeta_{\mathrm{A} 63-\mathrm{R} 80}$ heterodimers comprising the asymmetric unit.

The reduction in symmetry from a fourfold axis along $c$ in the tetragonal unit cell to a twofold axis in the orthorhombic unit cell, together with an increase in the number of molecules per asymmetric unit, helped us to identify the pseudo-merohedral twin operation $(k, h,-l)$ that accounted for the apparent fourfold Laue symmetry observed in the diffraction data. Consider a $P 2_{1} 2_{1} 2_{1}$ unit cell with unit-cell length $a$ approximately equal to unit-cell length $b$ (Fig. 4). Pseudomerohedral twinning can exchange the $a$ and $b$ axis under the twin relationship $(h, k, l) \rightarrow(k, h,-l)$, resulting in apparent tetragonal symmetry around the $c$ axis (Fig. $4 b$ ). The apparent symmetry observed in this case will be indistinguishable from a nontwinned $P 4_{3} 2_{1} 2$ (or $P 4_{1} 2_{1} 2$ ) unit cell (Fig. $4 a$ ), of which $P 2_{1} 2_{1} 2_{1}$ is a subgroup. Note that in this case the twinned $P 2{ }_{1} 2_{1} 2_{1}$ unit cell is less tightly packed, with one molecule per asymmetric unit (four per unit cell), than the corresponding nontwinned tetragonal $P 4_{3} 2_{1} 2$ (or $P 4_{1} 2_{1} 2$ ) cell, with one molecule per asymmetric unit (eight per unit cell). Based on the Matthews coefficient, we expected two molecules per asymmetric unit for the twinned $P 2_{1} 2_{1} 2_{1}$ unit cell. Note that the nontwinned crystals of the SIVmac239 $\mathrm{Nef}_{\text {core }}-\mathrm{TCR} \zeta_{\mathrm{DP} 1}$ complex, which did adopt true tetragonal symmetry with unitcell parameters similar to those of the twinned $P 2_{1} 2_{1} 2_{1}$ crystal, had a Matthews coefficient consistent with one molecule per asymmetric unit. Because the SIVmac239 $\mathrm{Nef}_{\text {core }}-\mathrm{TCR} \zeta_{\mathrm{DP} 1}$ and SIVmac239 $\mathrm{Nef}_{\text {core }}-\mathrm{TCR} \zeta_{\mathrm{A63}-\mathrm{R} 80}$ complexes had similar molecular sizes and crystallized in related unit cells with similar lengths and angles we expected similar packing, but this was inconsistent with the different packing expected for the related twinned $P 2_{1} 2_{1} 2_{1}$ and nontwinned $P 4_{3} 2_{1} 2$ (or $P 4_{1} 2_{1} 2$ ) unit cells shown in Fig. 4. Noncystallographic

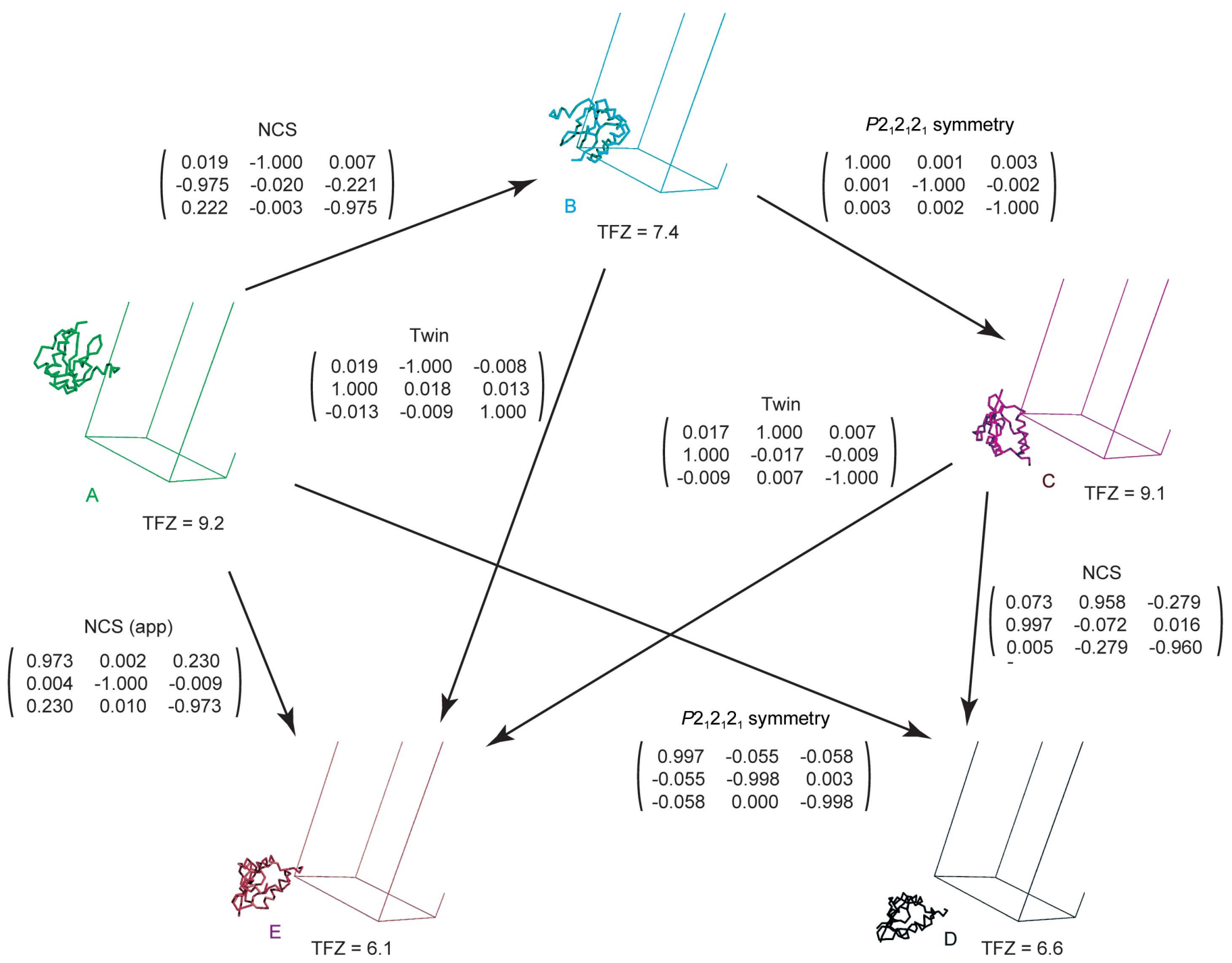

Figure 5

Molecular-replacement solutions. Five molecular-replacement solutions (A-E) are shown with their translation-function $Z$ (TFZ) scores denoted. The relationships and rotation matrices relating the molecular-replacement solutions are shown. 
symmetry relationships that are similar to crystallographic symmetry operators also can result in observed symmetry that is higher than that actually present in the crystal. For example, breakdown of the crystallographic fourfold axis in a tetragonal cell could result in an orthorhombic cell with pseudo-fourfold symmetry. In this case, the noncrystallographic symmetry relationship is similar to the missing crystallographic operator and the related tetragonal and orthorhombic unit cells would have similar packing (Fig. 4c). This arrangement can be particularly prone to pseudo-merohedral twinning as a result

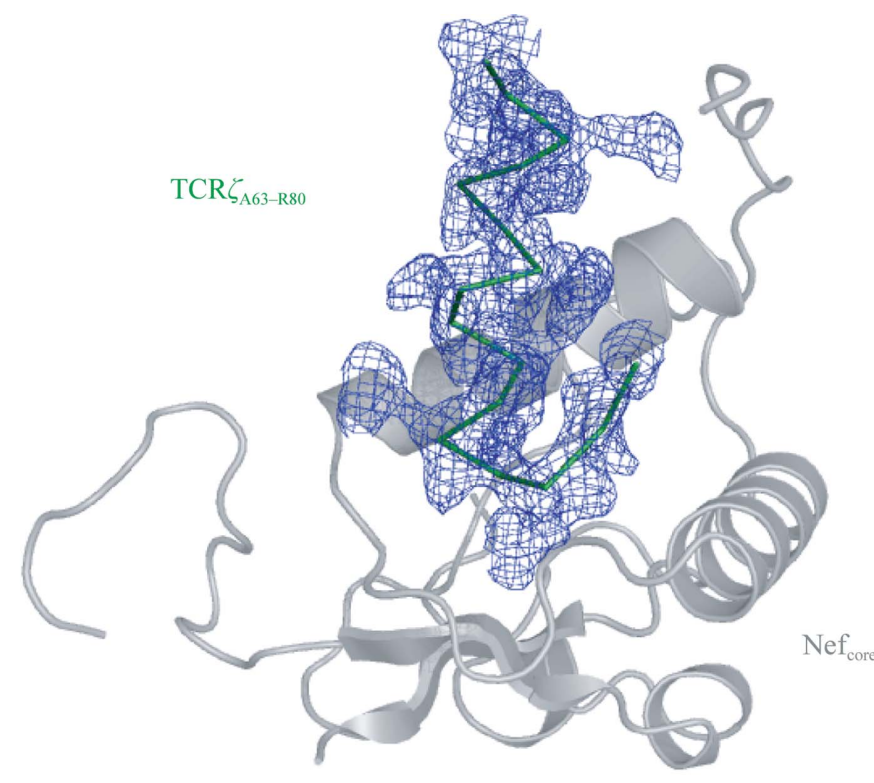

(a)

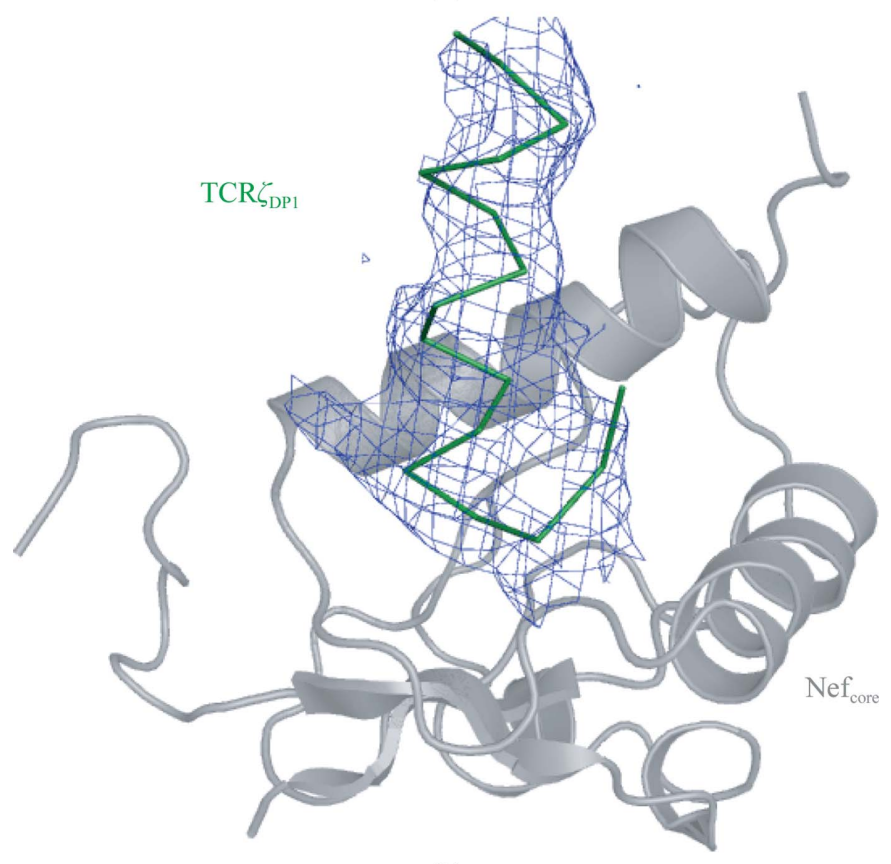

(b)

Figure 6

$2 F_{\mathrm{o}}-F_{\mathrm{c}}$ OMIT electron-density maps of the TCR $\zeta$ polypeptide. $2 F_{\mathrm{o}}-F_{\mathrm{c}}$ OMIT electron-density maps contoured at $1 \sigma$ calculated from the detwinned $P 2_{1} 2_{1} 2_{1}$ data of the SIVmac239 Nef $_{\text {core }}-\mathrm{TCR} \zeta_{\text {A63-R80 }}$ crystal (a) and the $P 4_{3} 2_{1} 2$ data of the SIVmac239 $\mathrm{Nef}_{\text {core }}-\mathrm{TCR} \zeta_{\mathrm{DP} 1}$ crystal (b) are shown for the region encompassing the TCR $\zeta$ polypeptide. of the similarity of the crystal packing along the $a$ and $b$ unitcell axes (Fig. 4c). We explored this scenario as an explanation for the observed twinning in the $P 2_{1} 2_{1} 2_{1}$ crystals with packing similar to nontwinned $P 4_{3} 2_{1} 2$ (or $P 4_{1} 2_{1} 2$ ) crystals.

\subsection{Structure determination and refinement of SIVmac239 $\mathrm{Nef}_{\text {core }}-\mathrm{TCR} \zeta_{\text {A63-R80 }}$ using twinned data}

After assignment of the SIVmac239 $\mathrm{Nef}_{\text {core }}-\mathrm{TCR} \zeta_{\mathrm{A} 63-\mathrm{R} 80}$ crystal data to the orthorhombic $P 2_{1} 2_{1} 2_{1}$ space group, several strong molecular-replacement solutions were readily found with TFZ scores of 6.1-9.2 using a consensus model derived from unliganded HIV-1 Nef $_{\text {core }}$ crystal structures. In principle, molecular-replacement solutions corresponding to both twin
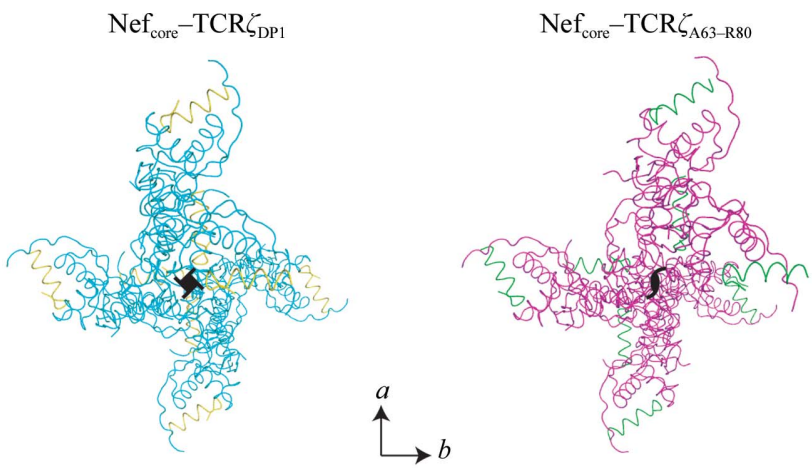

(a)

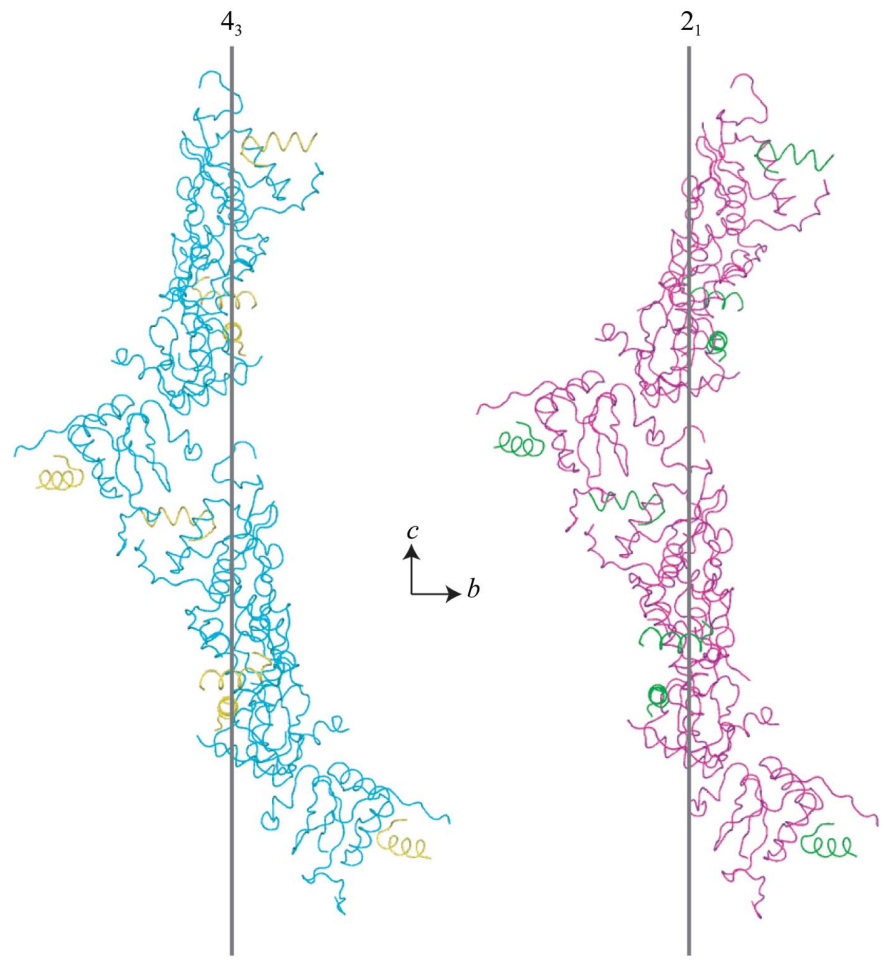

(b)

Figure 7

Crystal packing of the $P 4_{3} 2_{1} 2$ and $P 2_{1} 2_{1} 2_{1}$ crystal forms. (a) The crystal symmetry organization of the $P 4_{3} 2_{1} 2$ crystal form (left) and the $P 2_{1} 2_{1} 2_{1}$ crystal form (right) is shown viewed down the fourfold symmetry axis and the corresponding twofold symmetry axis for the two SIVmac239 $\mathrm{Nef}_{\text {core }}{ }^{-}$ $\mathrm{TCR} \zeta$ polypeptide complexes. In $(b)$ the crystal packing along the $c$ axis is shown for both crystal forms. SIVmac239 $\mathrm{Nef}_{\text {core }}$ and TCR $\zeta$ are colored cyan and yellow (left) and magenta and green (right), respectively. 
orientations and noncrystallographically related molecules are expected. Transformations among these solutions were examined to assign each to a twin or NCS domain (Fig. 5). All solutions could be accounted for using a single NCS transformation, the $(k, h,-l)$ twinning operator and the $P 2_{1} 2_{1} 2_{1}$ space-group symmetry. Two nearby molecules $(A$ and $B)$ in the same twin domain related by an approximate $90^{\circ}$ rotation were selected to comprise the asymmetric unit.

Once the twinning arrangement was properly understood and taken into account, model building and refinement in space group $P 2_{1} 2_{1} 2_{1}$ with two molecules per asymmetric unit was relatively straightforward. The twin operation $(k, h,-l)$ was factored into each round of twinned refinement in phenix.refine, which included three cycles of individual atomic displacement parameter ( $B$ factor) and energy-minimization refinement. The twin fraction $\alpha$ was also refined in each round and used to detwin the intensity data in order to generate
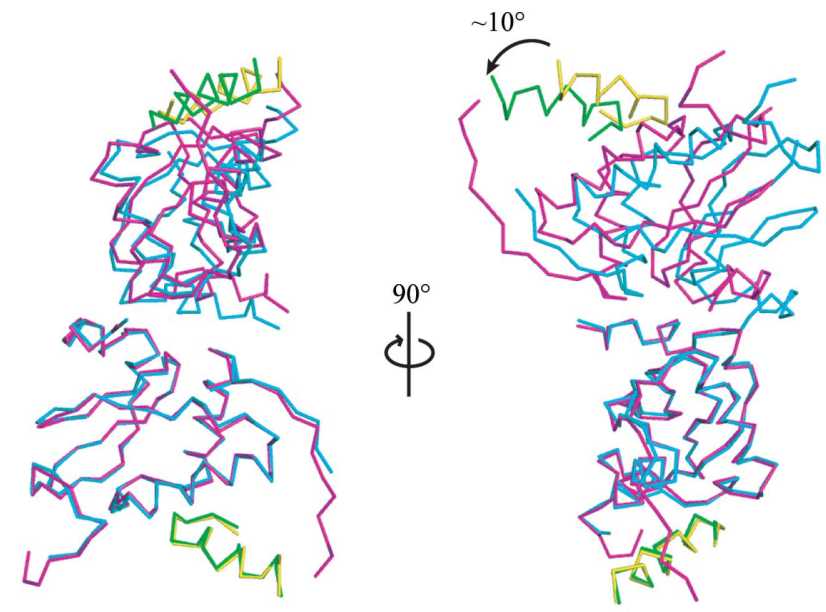

(a)

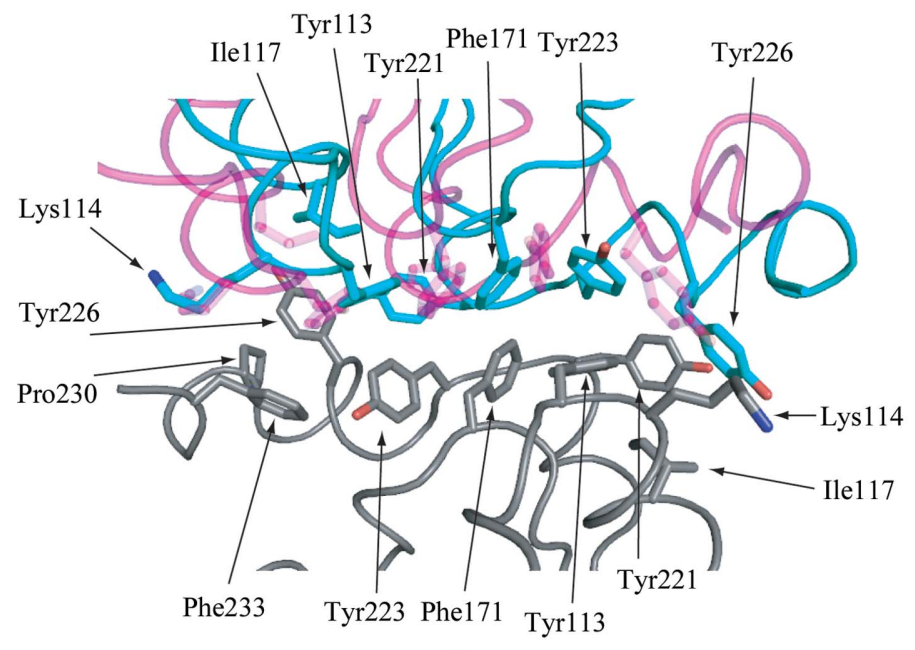

(b) interpretable $2 F_{\mathrm{o}}-F_{\mathrm{c}}$ and $F_{\mathrm{o}}-F_{\mathrm{c}}$ OMIT electron-density maps suitable for manual model building. However, during the first round of refinement, the twin fraction converged to 0.5 for crystal 1 and 0.426 for crystal 2 . The calculated $2 F_{\mathrm{o}}-F_{\mathrm{c}}$ electron-density maps generated by phenix.refine were noticeably less interpretable for crystal 1 than for crystal 2 . Therefore, structure determination proceeded with crystal 2 through iterative cycles of twinned refinement interspersed with rounds of model inspection and building. As the model and twin fraction continued to be refined, there was a marked improvement in the quality of the electron-density maps that allowed the building of five additional residues at the $\mathrm{N}$-terminus (Val98-Val102), one residue at the C-terminus (Gly234) and 11 residues in the internal disordered loop (Pro197-Trp207) of Nef; the starting model generated from the published crystal structures of HIV-1 Nef was missing nine residues at the $\mathrm{N}$-terminus, two residues at the $\mathrm{C}$-terminus and 29 residues in the disordered loop. Clear density for the $\mathrm{TCR} \zeta_{\mathrm{A} 63-\mathrm{R} 80}$ polypeptide ligand was observed and this region was also built into the structure, with 13 of the 16 resolved residues comprising a canonical $\alpha$-helix (Fig. 6). Water molecules were added to the model using the automated waterpicking functions in phenix.refine and Coot. The final structure (crystal 2) contained 120 residues of SIVmac239 Nef, 16 residues of TCR $\zeta, 116$ ordered water molecules and had $R_{\text {work }}$ and $R_{\text {free }}$ values of $17.0 \%$ and $18.4 \%$, respectively (Table 1 ).

\subsection{Structure determination and refinement of SIVmac239 $\mathrm{Nef}_{\text {core }}-\mathrm{TCR} \zeta_{\text {DP1 }}$}

With a high-resolution structure for SIVmac239 $\mathrm{Nef}_{\text {core }}{ }^{-}$ $\mathrm{TCR} \zeta_{\mathrm{A} 63-\mathrm{R} 80}$ structure in hand, we returned to the lowresolution nontwinned SIVmac239 $\mathrm{Nef}_{\text {core }}-\mathrm{TCR} \zeta_{\mathrm{DP} 1} P 4_{3} 2_{1} 2$ data set. In order to determine the low-resolution structure of

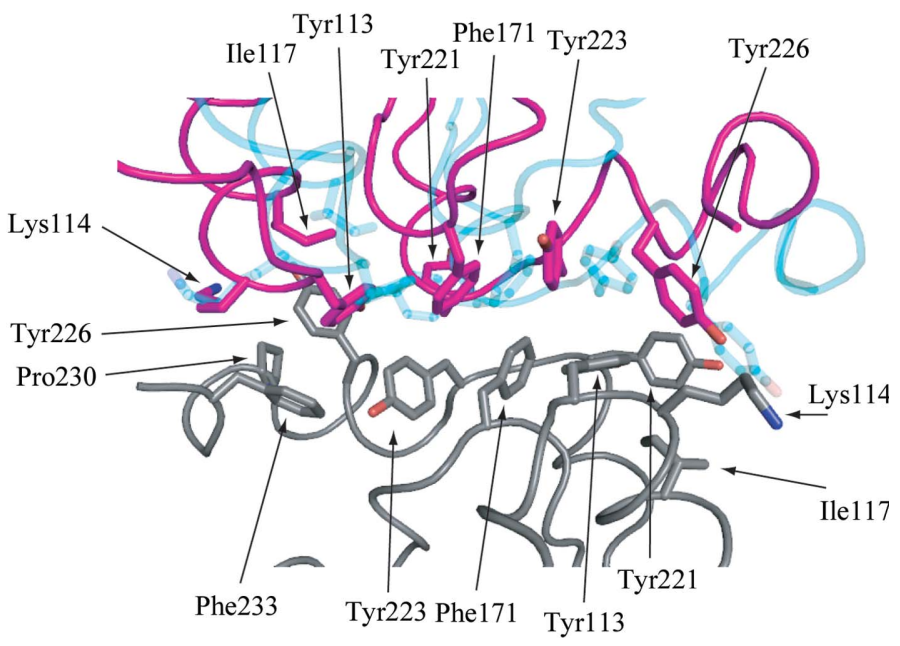

(c)

Figure 8

SIVmac239 $\mathrm{Nef}_{\text {core }}$ dimer interface in the $P 4_{3} 2_{1} 2$ and $P 2_{1} 2_{1} 2_{1}$ crystal forms. (a) Overlay of the two molecules in the asymmetric unit of the $P 2_{1} 2_{1} 2_{1}$ crystal (magenta/green) and two symmetry-related molecules $[(x, y, z),(y, x,-z)]$ in the $P 4_{3} 22_{1}$ crystal (cyan/yellow). The SIVmac 239 Nef core is colored magenta $\left(P 2_{1} 2_{1} 2_{1}\right.$ crystal) or cyan $\left(P 4_{3} 2_{1} 2\right.$ crystal) and the TCR $\zeta$ polypeptide is colored green $\left(P 2_{1} 2_{1} 2_{1}\right.$ crystal) or yellow $\left(P 4_{3} 2_{1} 2\right.$ crystal). The structures of the lower SIVmac239 $\mathrm{Nef}_{\text {core }}-\mathrm{TCR} \zeta$ polypeptide complexes were aligned by least-squares methods. The relative $10^{\circ}$ counterclockwise rotation of the top

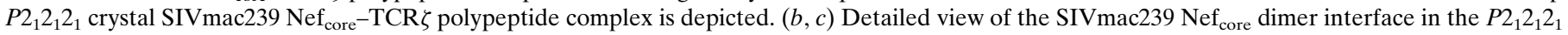
(magenta) and $P 4_{3} 2_{1} 2$ (blue) crystals. The aligned lower SIVmac239 $\mathrm{Nef}_{\text {core }}-\mathrm{TCR} \zeta$ polypeptide complex is colored grey and the side chains of residues involved in the interface are shown as stick models. (b) $P 2_{1} 2_{1} 2_{1}$ crystal form (cyan) is highlighted. (c) $P 4_{3} 2_{1} 2$ crystal form (magenta) is highlighted. 
SIVmac239 $\mathrm{Nef}_{\text {core }}-\mathrm{TCR} \zeta_{\mathrm{DP} 1}$, the high-resolution SIVmac239 $\mathrm{Nef}_{\text {core }}-\mathrm{TCR} \zeta_{\mathrm{A} 63-\mathrm{R} 80}$ structure was used as the starting model for refinement and building. Structure determination by molecular replacement was repeated for the nontwinned SIVmac239 $\mathrm{Nef}_{\text {core }}-\mathrm{TCR} \zeta_{\text {DP1 }}$ data. A stronger molecularreplacement solution was found $(\mathrm{TFZ}=10.5, \mathrm{LLG}=266)$ but in the same general orientation as that described previously. Refinement of atomic positions and individual $B$ factors was performed in phenix.refine as described above for the twinned crystal, although without the twin-refinement and detwinning steps. The TCR $\zeta_{\text {DP1 }}$ peptide extends 12 residues futher at the $\mathrm{N}$-terminus and 14 residues further at the $\mathrm{C}$-terminus compared with the TCR $\zeta_{\mathrm{A63}-\mathrm{R} 80}$ polypeptide, but no additional electron density was observed beyond that seen in the SIVmac239 $\mathrm{Nef}_{\text {core }}-\mathrm{TCR} \zeta_{\mathrm{A} 63-\mathrm{R} 80}$ complex (Fig. 6), suggesting that both the $\mathrm{N}$ - and $\mathrm{C}$-termini of the TCR $\zeta_{\mathrm{DP} 1}$ fragment were disordered and that no additional Nef contacts were present. The final structure of the SIVmac239 $\mathrm{Nef}_{\text {core }}-\mathrm{TCR} \zeta_{\mathrm{DP} 1}$ complex contained 111 residues of Nef and 16 residues of TCR $\zeta$ and had $R_{\text {work }}$ and $R_{\text {free }}$ values of $30.1 \%$ and $32.9 \%$, respectively (Table 1$)$.

\subsection{Analysis of the $P 2_{1} 2_{1} 2_{1}$ and $P 4_{3} 2_{1} 2$ crystal forms of the SIVmac239 Nef $_{\text {core }}-$ TCR $\zeta$ polypeptide complex}

As described above, the SIVmac239 $\mathrm{Nef}_{\text {core }}-\mathrm{TCR} \zeta$ polypeptide complex crystallized in two related but different crystal lattices depending on the length of the TCR $\zeta$ ligand. In the presence of the longer 43-residue TCR $\zeta_{\mathrm{DP} 1}$ polypeptide SIVmac239 $\mathrm{Nef}_{\text {core }}$ crystallized in the tetragonal $P 4_{3} 2_{1} 2$ space group with one SIVmac239 $\mathrm{Nef}_{\text {core }}-\mathrm{TCR} \zeta_{\mathrm{DP} 1}$ heterodimer comprising the asymmetric unit. In the presence of the shorter 18-residue $\mathrm{TCR} \zeta_{\mathrm{A} 63-\mathrm{R} 80}$ polypeptide the complex unexpectedly crystallized in the $P 2_{1} 2_{1} 2_{1}$ space group with severe pseudo-hemihedral twinning. In this crystal form, a rotation

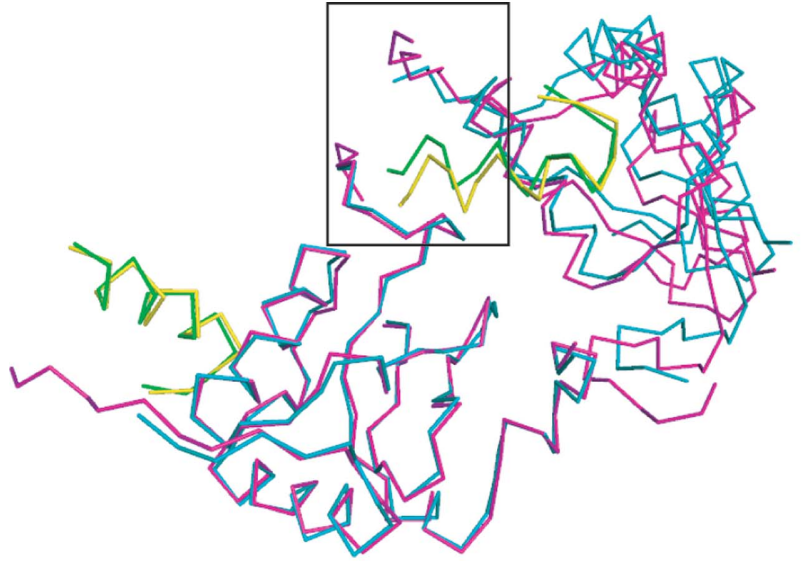

(a)

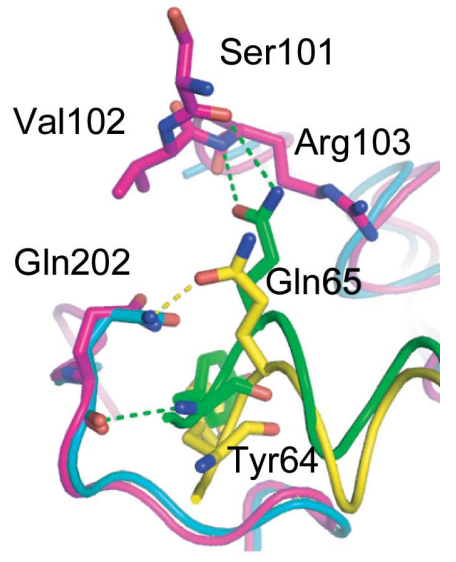

(b)
Figure 9

Variation in the crystal contact hydrogen-bond network. Overlay of the crystal-packing interface between two asymmetric units of the $P 2{ }_{1} 2_{1} 2_{1}$ crystal lattice (SIVmac239 Nef is shown in magenta and TCR $\zeta$ is shown in green) and two symmetry-related molecules $(y, x,-z)$ and $(1 / 2+y, 1 / 2-x, 1 / 4+z)$ of the $P 4_{3} 2_{1} 2$ crystal lattice (SIVmac239 Nef is shown in cyan and TCR $\zeta$ is shown in yellow). Alignment was performed by leastsquares methods using one SIVmac239 Nef-TCR $\zeta$ polypeptide complex [at the bottom in $(a)$ ]. Hydrogen bonds present in the crystal lattices are represented by dashed lines and are colored green and yellow for the $P 2_{1} 2_{1} 2_{1}$ and $P 4_{3} 2_{1} 2$ crystal forms, respectively. axis parallel to $c$ exhibited pseudo-fourfold symmetry that deviated slightly from the crystallographic fourfold screw axis observed in the $P_{3} 2_{1} 2$ crystal form (Fig. 7). The overall packing of the unit cell was also condensed in the orthorhombic crystal form, as evidenced by an $\sim 4 \AA \quad(\sim \%)$ reduction in the $a$ and $b$ axes and an $\sim 6 \AA(\sim 3 \%)$ shortening of the $c$ axis.

The transformation from the tetragonal to the orthorhombic crystal system was caused by the introduction of noncrystallographic symmetry (NCS) and rearrangement of the hydrogen-bonding network at the crystal contact sites. The $P 4_{3} 2_{1} 2$ crystal form contained one molecule per asymmetric unit. The $P 2_{1} 2_{1} 2_{1}$ crystal form contained two molecules per asymmetric unit which were no longer related by a crystallographic twofold symmetry operation $(y, x,-z)$ but instead by a twofold NCS operation,

$$
\left(\begin{array}{ccc}
0.000 & 1.000 & 0.000 \\
1.000 & 0.000 & 0.000 \\
0.000 & 0.000 & -1.000
\end{array}\right) \rightarrow\left(\begin{array}{ccc}
0.036 & 0.999 & -0.027 \\
0.981 & -0.030 & 0.194 \\
0.193 & -0.033 & -0.981
\end{array}\right) \text {. }
$$

In the tetragonal crystal form the SIVmac239 $\mathrm{Nef}_{\text {core }}{ }^{-}$ $\mathrm{TCR} \zeta_{\mathrm{DP} 1}$ complex and its symmetry-related partner $(y, x,-z)$ form an antiparallel dimer similar to the crystallographic dimer described previously for the HIV-1 Nef core $_{\text {(Arold } \text { et al., }}$ 1997). Structural alignment of one SIVmac239 $\mathrm{Nef}_{\text {core }}{ }^{-}$ TCR $\zeta_{\text {A63-R80 }}$ complex from the $P 2_{1} 2_{1} 2_{1}$ crystal form with its corresponding molecule in the $P_{3} 2_{1} 2$ crystallographic dimer reveals that the NCS-related molecule in the orthorhombic crystal form is rotated by $\sim 10^{\circ}$ from its corresponding molecule in the $P 4_{3} 2_{1} 2$ crystal form (Fig. 8). The interface between the two molecules involves the C-terminus of SIVmac239 $\mathrm{Nef}_{\text {core }}$ and is predominantly occupied by aromatic residues (Tyr113, Tyr221, Phe171, Tyr223 and Tyr226). As shown in Fig. 8(b), SIVmac239 Nef $_{\text {core }}$ is rotated as a single rigid body in the orthorhombic crystal form with no significant changes in either main-chain or side-chain geometry, suggesting that the crystallographic $\mathrm{Nef}_{\text {core }}$ dimer interface is flexible and permissible to variations in crystal packing.

Alternate crystal packing was also observed at the crystal contact of two asymmetric units in the orthorhombic crystal form and the corresponding symmetryrelated molecules $(y, x,-z)$ and $(1 / 2+y, 1 / 2-x, 1 / 4+z)$ in the tetragonal crystal form. The interface involves three proteins: SIVmac239 $\mathrm{Nef}_{\text {core }}$ and its bound $\mathrm{TCR} \zeta$ polypeptide ligand from the symmetry-related molecule 
$(1 / 2+y, 1 / 2-x, 1 / 4+z)$ and SIVmac239 $\mathrm{Nef}_{\text {core }}$ from the symmetry-related molecule $(y, x,-z)$ (Fig. $9 a)$. Interestingly, the $\mathrm{N}$-terminus of the TCR $\zeta$ polypeptide abuts the neighboring SIVmac239 $\mathrm{Nef}_{\text {core }}$ protein, suggesting that the length of the N-terminal sequence of the TCR $\zeta$ polypeptide ligand directs the space group in which the SIVmac239 $\mathrm{Nef}_{\text {core }}-\mathrm{TCR} \zeta$ polypeptide complex crystals grow. Superimposition of the $\mathrm{TCR} \zeta$ polypeptide helix from the symmetry-related molecule $(1 / 2+y, 1 / 2-x, 1 / 4+z)$ with its corresponding partner in the $P 2{ }_{1} 2_{1} 2_{1}$ crystal form reveals that the neighboring SIVmac239 $\mathrm{Nef}_{\text {core }}$ protein is rotated $\sim 4.5^{\circ}$ inwards towards the pseudofourfold symmetry axis in the $P 2_{1} 2_{1} 2_{1}$ crystal form (Fig. $9 a$ ).

Accompanying the transformation is a possible reorganization of the hydrogen-bonding network at the crystal contact site. In the orthorhombic crystal form the TCR $\zeta_{\mathrm{A} 63-\mathrm{R} 80}$ polypeptide forms a main-chain hydrogen bond to the neighboring SIVmac239 Nef $_{\text {core }}$ protein between the main-chain amide of TCR $\zeta$ Tyr64 and the side-chain carbonyl of Nef Gln202 (Fig. 9b). TCR $\zeta$ residue Gln65 additionally participates in hydrogen bonding to the main-chain amide and carbonyl of residues Arg103 and Val102, respectively, on its bound SIVmac239 $\mathrm{Nef}_{\text {core }}$ partner. Interestingly, this interaction orders the proline-rich region in the N-terminus of the bound SIVmac239 $\mathrm{Nef}_{\text {core }}$ into a polyproline type II (PPII) helix as evidenced by the clearly resolved electron-density maps calculated from the $P 2_{1} 2_{1} 2_{1}$ crystal data for that region. This carries significant functional importance owing to the regulatory role that the PPII helix on HIV-1 Nef has been suggested to play in modulating kinase activity through its interaction with the SH3 domain of the kinase (Arold et al., 1997; Lee et al., 1996). The PPII helix was found to be disordered in the unliganded HIV-1 Nef core crystals and was only ordered in crystals containing the Fyn SH3 domain. Surprisingly, the hydrogen-bonding network between the TCR $\zeta$ polypeptide and its bound SIVmac239 $\mathrm{Nef}_{\text {core }}$ partner is seemingly absent in the tetragonal crystal form; this explains the lack of electron density calculated from the $\mathrm{P}_{3} 2_{1} 2$ data for the $\mathrm{N}$-terminus of SIVmac239 $\mathrm{Nef}_{\text {core }}$ since the PPII helix would no longer be expected to be ordered. Instead of participating in a sidechain-main-chain hydrogen bond with its bound partner, residue Gln65 in TCR $\zeta$ is translocated in the tetragonal crystal form, bringing it into close enough proximity to residue Gln202 on the neighboring SIVmac239 Nef $_{\text {core }}$ protein to participate in a side-chain-side-chain hydrogen bond. The main-chain-main-chain hydrogen bond between the TCR $\zeta$ polypeptide and the neighboring SIVmac239 $\mathrm{Nef}_{\text {core }}$ protein is also lost in the rearranged $P 4_{3} 2_{1} 2$ crystal contact interface.

Since the proposed hydrogen bond between Gln65 on TCR $\zeta$ and Gln202 on SIVmac239 was formed by TCR $\zeta$ and an adjacent SIVmac239 $\mathrm{Nef}_{\text {core }}$ protein in the crystal lattice and not its interacting SIVmac239 $\mathrm{Nef}_{\text {core }}$ partner, it is likely to be an artifact of crystallization that was necessary for proper lattice packing in the tetragonal crystal form. Curiously, the more physiologically relevant interaction of Gln65 on TCR $\zeta$ with its bound SIVmac239 $\mathrm{Nef}_{\text {core }}$ partner was restored when the TCR $\zeta$ polypeptide was truncated. The loss of the crystal contact hydrogen bond reduced the crystal symmetry to an orthorhombic crystal lattice that was subsequently prone to twinning. This was unexpected owing to the inclusion of a more complete TCR $\zeta$ sequence in the tetragonal crystal and represents an interesting scenario in which a protein-ligand interaction was disrupted by a crystal contact interaction that permitted higher order crystal packing.

\section{Conclusions}

Crystal twinning can be induced by a number of perturbations, including heavy-metal soaking, ligand binding, selenomethionine substitution, flash-freezing and the introduction of point mutations (Parsons, 2003; Helliwell et al., 2006). The structure determination of the two SIVmac239 $\mathrm{Nef}_{\text {core }}-\mathrm{TCR} \zeta$ polypeptide complexes provides a unique example of crystal twinning caused by the modification of peptide-ligand size. Truncation of the TCR $\zeta$ polypeptide reduced the crystal symmetry from a tetragonal crystal system to an orthorhombic crystal system and introduced an NCS operation that only deviated slightly from the true fourfold symmetry axis. The pseudo-symmetry in the $P 2_{1} 2_{1} 2_{1}$ crystal made crystal growth highly susceptible to crystal twinning but serendipitously restored a physiologically relevant protein-ligand interaction at the crystal contact interface.

This work was supported by grant R21-AI074616 from the National Institutes of Health. Use of the National Synchrotron Light Source, Brookhaven National Laboratory was supported by the US Department of Energy, Office of Science, Office of Basic Energy Sciences under Contract No. DEAC02-98CH10886. We would like to thank Alexei Soares and Howard Robinson at beamline X29 (NSLS) for technical assistance and Tina Nguyen, Eric Schreiter and Zarixia Zavala-Ruiz for helpful discussions.

\section{References}

Adams, P. D., Grosse-Kunstleve, R. W., Hung, L.-W., Ioerger, T. R., McCoy, A. J., Moriarty, N. W., Read, R. J., Sacchettini, J. C., Sauter, N. K. \& Terwilliger, T. C. (2002). Acta Cryst. D58, 1948-1954.

Arien, K. K. \& Verhasselt, B. (2008). Curr. HIV Res. 6, 200-208.

Arold, S., Franken, P., Strub, M. P., Hoh, F., Benichou, S., Benarous, R. \& Dumas, C. (1997). Structure, 5, 1361-1372.

Bell, I., Ashman, C., Maughan, J., Hooker, E., Cook, F. \& Reinhart, T. A. (1998). J. Gen. Virol. 79, 2717-2727.

Britton, D. (1972). Acta Cryst. A28, 296-297.

Brooks, C. L., Blackler, R. J., Gerstenbruch, S., Kosma, P., MüllerLoennies, S., Brade, H. \& Evans, S. V. (2008). Acta Cryst. D64, $1250-1258$.

Collaborative Computational Project, Number 4 (1994). Acta Cryst. D50, 760-763.

Emsley, P. \& Cowtan, K. (2004). Acta Cryst. D60, 2126-2132.

Fackler, O. T., Wolf, D., Weber, H. O., Laffert, B., D'Aloja, P., SchulerThurner, B., Geffin, R., Saksela, K., Geyer, M., Peterlin, B. M., Schuler, G. \& Baur, A. S. (2001). Curr. Biol. 11, 1294-1299.

Fenard, D., Yonemoto, W., de Noronha, C., Cavrois, M., Williams, S. A. \& Greene, W. C. (2005). J. Immunol. 175, 6050-6057.

Franken, P., Arold, S., Padilla, A., Bodeus, M., Hoh, F., Strub, M. P., Boyer, M., Jullien, M., Benarous, R. \& Dumas, C. (1997). Protein Sci. 6, 2681-2683. 
Govindasamy, L., Reutzel, R., Agbandje-McKenna, M. \& McKenna, R. (2004). Acta Cryst. D60, 1040-1047.

Grainger, C. T. (1969). Acta Cryst. A25, 427-434.

Grzesiek, S., Bax, A., Hu, J. S., Kaufman, J., Palmer, I., Stahl, S. J., Tjandra, N. \& Wingfield, P. T. (1997). Protein Sci. 6, 1248-1263.

Helliwell, M., Collison, D., John, G. H., May, I., Sarsfield, M. J., Sharrad, C. A. \& Sutton, A. D. (2006). Acta Cryst. B62, 68-85.

Howe, A. Y., Jung, J. U. \& Desrosiers, R. C. (1998). J. Virol. 72, $9827-$ 9834.

Landon, M. (1977). Methods Enzymol. 47, 145-149.

Larsen, N. A., Heine, A., de Prada, P., Redwan, E.-R., Yeates, T. O., Landry, D. W. \& Wilson, I. A. (2002). Acta Cryst. D58, 2055-2059.

Laskowski, R. A., Moss, D. S. \& Thornton, J. M. (1993). J. Mol. Biol. 231, 1049-1067.

Lee, C. H., Saksela, K., Mirza, U. A., Chait, B. T. \& Kuriyan, J. (1996). Cell, 85, 931-942.

Lovell, S. C., Davis, I. W., Arendall, W. B. III, de Bakker, P. I., Word, J. M., Prisant, M. G., Richardson, J. S. \& Richardson, D. C. (2003). Proteins, 50, 437-450.

Matthews, B. W. (1968). J. Mol. Biol. 33, 491-497.

McPherson, A. (1982). The Preparation and Analysis of Protein Crystals. New York: John Wiley.

Munch, J., Janardhan, A., Stolte, N., Stahl-Hennig, C., Ten Haaft, P., Heeney, J. L., Swigut, T., Kirchhoff, F. \& Skowronski, J. (2002). J. Virol. 76, 12360-12364.

Otwinowski, Z. \& Minor, W. (1997). Methods Enzymol. 276, 307-326. Padilla, J. E. \& Yeates, T. O. (2003). Acta Cryst. D59, 1124-1130.

Parsons, S. (2003). Acta Cryst. D59, 1995-2003.
Peter, F. (1998). Immunity, 9, 433-437.

Rudolph, M. G., Wingren, C., Crowley, M. P., Chien, Y. \& Wilson, I. A. (2004). Acta Cryst. D60, 656-664.

Schaefer, T. M., Bell, I., Fallert, B. A. \& Reinhart, T. A. (2000). J. Virol. 74, 3273-3283.

Schaefer, T. M., Bell, I., Pfeifer, M. E., Ghosh, M., Trible, R. P., Fuller, C. L., Ashman, C. \& Reinhart, T. A. (2002). Virology, 302, 106-122. Schindler, M. et al. (2006). Cell, 125, 1055-1067.

Sigalov, A., Aivazian, D. \& Stern, L. (2004). Biochemistry, 43, 2049 2061.

Sigalov, A. B., Kim, W. M., Saline, M. \& Stern, L. J. (2008). Biochemistry, 47, 12942-12944.

Stein, N. (2008). J. Appl. Cryst. 41, 641-643.

Storoni, L. C., McCoy, A. J. \& Read, R. J. (2004). Acta Cryst. D60, 432-438.

Swigut, T., Greenberg, M. \& Skowronski, J. (2003). J. Virol. 77, 81168126.

Swigut, T., Iafrate, A. J., Muench, J., Kirchhoff, F. \& Skowronski, J. (2000). J. Virol. 74, 5691-5701.

Thoulouze, M. I., Sol-Foulon, N., Blanchet, F., Dautry-Varsat, A., Schwartz, O. \& Alcover, A. (2006). Immunity, 24, 547-561.

Willard-Gallo, K. E., Furtado, M., Burny, A. \& Wolinsky, S. M. (2001). Eur. J. Immunol. 31, 969-979.

Xu, X. N., Laffert, B., Screaton, G. R., Kraft, M., Wolf, D., Kolanus, W., Mongkolsapay, J., McMichael, A. J. \& Baur, A. S. (1999). J. Exp. Med. 189, 1489-1496.

Yeates, T. O. (1988). Acta Cryst. A44, 142-144.

Yeates, T. O. (1997). Methods Enzymol. 276, 344-358. 